anales de psicología, 2014, vol. 30, nº 3 (octubre), 1044-1060 http://dx.doi.org/10.6018/analesps.30.3.138981
(C) Copyright 2014: Servicio de Publicaciones de la Universidad de Murcia. Murcia (España) ISSN edición impresa: 0212-9728. ISSN edición web (http://revistas.um.es/analesps): 1695-2294

\title{
Análisis de los estereotipos de género actuales
}

\author{
Rosario Castillo-Mayén ${ }^{1 *}$ y Beatriz Montes-Berges ${ }^{2}$ \\ ${ }^{1}$ University College Dublin \\ ${ }^{2}$ Universidad de Jaén
}

\begin{abstract}
Resumen: Los estereotipos de género son creencias sobre las características asociadas a mujeres y hombres que mantienen la discriminación de género. El estudio de los estereotipos que prevalecen en nuestros días se torna indispensable para conocer los cambios que se van produciendo en relación a este fenómeno. Con este objetivo, se construyó una escala que contenía 258 características estereotípicas con dos versiones que permitieran conocer por separado cómo se percibe actualmente a cada género. Una muestra de 164 estudiantes de universidad (50\% mujeres) completaron ambas versiones. Los resultados de este estudio permiten identificar los estereotipos de género vigentes señalando aquéllos que en la actualidad aún se asignan diferencialmente a cada género e independientemente de características sociodemográficas como la edad o el sexo. Además, se recogen los estereotipos de género que han surgido nuevos. Destacamos los importantes cambios que se han producido en este tipo de creencias, especialmente en los estereotipos femeninos. Según la teoría del rol social, tales cambios serían una consecuencia del cambio producido en los roles sociales. Las conclusiones subrayan que, si bien parte de los resultados suponen un avance en el logro de la igualdad, aún se atribuyen características estereotípicas tradicionales a cada género que perpetúan la discriminación.
\end{abstract}

Palabras clave: estereotipos de género; discriminación de género; dinámica de los estereotipos; roles sociales.

\section{Introducción}

Existe un amplio acuerdo en considerar a los estereotipos en general como el aspecto cognitivo del prejuicio (Dovidio, Evans y Tyler, 1986). Los estereotipos influyen en el procesamiento de la información sobre los grupos sociales (Dovidio et al., 1986), así como sobre nuestro comportamiento y el de los demás (ej., Heilman, 2001; Steele, 1997). Centrándonos en los estereotipos de género, estos son uno de los tipos de creencias que, junto con la identidad e ideología de género, subyacen a las conductas discriminatorias basadas en la categorización de una persona como mujer u hombre (Barberá, 1998; Moya y Puertas, 2003). Por estereotipos de género se entiende el conjunto estructurado de creencias compartidas dentro de una cultura o grupo acerca de los atributos o características que posee cada género (Moya, 2003). Según la teoría del rol social (Eagly, 1987; Eagly, Wood y Diekman, 2000; Eagly, Wood y JohannesenSchmidt, 2004), las creencias estereotípicas sobre los grupos de género surgen porque al observar que cada grupo realiza roles sociales diferentes se infiere la existencia de disposiciones internas distintas. Estas creencias, el proceso de socialización y los procesos individuales favorecen la aparición de comportamientos diferenciados entre mujeres y hombres, $\mathrm{y}$ en consecuencia el mantenimiento de estos estereotipos. Además, los estereotipos de género conllevan importantes consecuencias negativas pues limitan el desarrollo integral de

* Dirección para correspondencia [Correspondence address]: Rosario Castillo-Mayén. UCD Sutherland School of Law. University College Dublin. Belfield, Dublin 4, Ireland.

E-mail: rosario.castillomayen@gmail.com

\section{Title: Analysis of current gender stereotypes.}

Abstract: Gender stereotypes are beliefs about attributes associated to women and men that reveal gender discrimination. In order to identify changes of gender discrimination, the study of the stereotypes that prevail nowadays is essential. With this in mind, a scale consisting of 258 stereotypic characteristics was elaborated. This scale comprised two versions, one for female and one for male, which permits the understanding of how each gender is perceived currently. Both versions were filled out by 164 undergraduates (50\% women). Taking into account those stereotypes that are still differentially assigned to each gender, this study identifies current gender stereotypes that are independent of sociodemographic characteristics, such as age or sex. In addition, new gender stereotypes emerged recently were gathered, and important changes of stereotypes were emphasized, especially those of feminine stereotypes. According to social role theory, these changes are the consequence of social roles changes. Conclusions highlight that, although part of the results involve progress on the achievement of equality, traditional stereotypic characteristics are still referred to each gender, which perpetuate discrimination.

Key words: gender stereotypes; gender discrimination; dynamic of stereotypes; social roles. las personas, influyendo sobre sus preferencias, desarrollo de habilidades, aspiraciones, emociones, estado físico, rendimiento, etc. Estas consecuencias recaen indudablemente en mayor medida sobre las mujeres, favoreciendo además, su vulnerabilidad para ser víctimas de violencia. Dada la importancia de los estereotipos de género en los procesos discriminatorios, resulta esencial saber si el contenido de estos estereotipos permanece estable o si se ha producido algún cambio. En este estudio, nos proponemos identificar los estereotipos de género que se mantienen vigentes en la actualidad, es decir, pretendemos señalar aquellas características que se asignan de manera diferencial a cada grupo de género.

El contenido de los estereotipos de género es multifacético, si bien su estudio se realiza principalmente a partir de los rasgos de personalidad, en los que se distinguen las dimensiones fundamentales de instrumentalidad y expresividad (ej., Bem, 1974; López-Sáez y Morales, 1995; Spence, Helmreich y Stapp, 1974) o de agente y comunalidad (ej., Eagly, 1987; Glick y Fiske, 2001). Generalmente, tanto la hetero-percepción como auto-percepción de estos rasgos coincide con que los atributos considerados instrumentales o agentes (ej., independiente, asertivo, autoeficaz, orientado al logro) se asocian más a los hombres, mientras que los atributos expresivos o comunales (ej., cariñosa, afectuosa, amable, orientada a los demás) se asocian más a las mujeres. Además, los estereotipos de género contienen un carácter tanto descriptivo (describen cómo son los grupos de género) como prescriptivo (indican cómo deben ser estos grupos), este último en mayor medida que otros grupos sociales (ej., Burgess y Borgida, 1999; Eagly, 1987; Fiske y Stevens, 1993). Las normas prescriptivas sobre los grupos de género produ- 
cen severas consecuencias sobre su aceptación y posible cambio (ej., López-Sáez y Lisbona, 2009). Así, la diferenciación estereotípica de hombres y mujeres conlleva importantes consecuencias para el mantenimiento del sistema basado en el género (Jackman, 1994). Por un lado, se favorece la naturalización de las diferencias, tratando a cada grupo como si realmente fuera más apropiado para ocupar los roles prescritos por la sociedad (Hoffman y Hurst, 1990; Jost y Hamilton, 2005). Por otro lado, el carácter aparentemente favorable del estereotipo femenino y la valoración positiva que la sociedad realiza de algunos roles asociados con las mujeres (ej., cuidar de la familia), dificulta que éstas rechacen dicho sistema.

Aunque el contenido de los estereotipos de género se encuentra ampliamente compartido por diferentes culturas (ej., Williams y Best, 1982, 1990), y a pesar de que su carácter prescriptivo favorece el mantenimiento de los mismos, diferentes investigaciones han analizado la dinámica de estas creencias observando su evolución en diferentes momentos temporales, así como la influencia de diferentes características sociales y culturales en su estabilidad (ej., Castillo-Mayén y Montes-Berges, 2007; Diekman y Eagly, 2000; Diekman, Eagly, Mladinic y Ferreira 2005; López-Sáez, Morales y Lisbona, 2008; López-Zafra, García-Retamero, Diekman y Eagly, 2008; Montes-Berges, 2002; Moya y Pérez, 1990; Spence y Buckner, 2000). Según la teoría del rol social (Eagly, 1987; Eagly et al., 2000; Eagly et al., 2004), se espera que un cambio en los roles sociales se traduzca en un cambio en los estereotipos asignados a mujeres y hombres. La mayoría de estudios realizados en este ámbito confirman, al menos parcialmente, los postulados de esta teoría, poniendo de manifiesto una mayor dinámica en el estereotipo femenino (ej., Diekman y Eagly, 2000; García-Retamero, Müller y López-Zafra, 2009; López-Sáez et al., 2008). Este mayor cambio en los estereotipos asignados a las mujeres se explica porque las mujeres han experimentado, a su vez, un mayor cambio en los roles sociales que ocupan en comparación con los hombres (Sczesny, Bosak, Diekman y Twenge, 2008).

Los estudios que recientemente han analizado la influencia concreta de diferentes variables socioculturales y sociodemográficas (ej., Castillo-Mayén y Montes-Berges, 2007; Diekman y Eagly, 2000; Diekman et al., 2005; GarcíaRetamero et al., 2009; López-Sáez y Lisbona, 2009; RochaSánchez y Díaz-Loving, 2005; Wilde y Diekman, 2005), indican, por ejemplo, que el tamaño poblacional incide sobre la aplicación de los estereotipos tradicionales (García-Retamero et al., 2009), o que la edad, sexo y nivel educativo influyen sobre la vigencia de la dimensión prescriptiva de los estereotipos de género, pero no sobre la dimensión descriptiva (López-Sáez y Lisbona, 2009).

El procedimiento habitual en la mayoría de estudios que analizan la estabilidad de los estereotipos de género consiste en presentar un listado de estereotipos que tradicionalmente se han considerado como característicos de uno u otro grupo de género. Sin embargo, este procedimiento puede impedir que se conozca si la evolución de estos estereotipos ha dado lugar a que en la actualidad se incluyan otras características no consideradas hasta ahora estereotípicas. Con la intención de identificar el surgimiento de nuevos estereotipos, en este estudio se utilizó un amplio listado de adjetivos que incluía tanto estereotipos de género tradicionales como otros estereotipos que comúnmente se asocian a hombres o mujeres aunque no suelan incluirse en las investigaciones (ej., "decididos" y "fanfarrones" para los hombres, o "solidarias" y "superficiales" para las mujeres). La identificación de nuevos estereotipos permitiría enriquecer los resultados obtenidos hasta ahora en este ámbito de investigación. Además, teniendo en cuenta los resultados de las investigaciones sobre la dinámica de los estereotipos de género, en las que se observa el efecto de distintas variables sobre su vigencia, en este estudio nos proponemos identificar aquellos estereotipos que en la actualidad se asignan diferencialmente a los grupos de género y cuya percepción como vigentes no se deba al efecto de ninguna característica sociodemográfica. Para ello, como variables sociodemográficas se tendrán en cuenta algunas de las utilizadas en otros estudios, concretamente el sexo de los participantes, su edad, estado civil, orientación política y religiosidad. De esta forma, este estudio nos permitirá conocer los estereotipos sobre los que actualmente existe un acuerdo en ser considerados como más característicos de uno u otro género, independientemente de las características sociodemográficas de los participantes.

Concretamente, con la finalidad de identificar los estereotipos de género actuales, en primer lugar, se elaboró una amplia escala de adjetivos que contenía tanto los estereotipos de género tradicionales procedentes de la literatura más relevante en este ámbito como otras características que también se suelen utilizar para describir a los grupos de género. De esta escala se realizaron dos versiones que se administraron conjuntamente y de forma contrabalanceada, una para indicar en qué medida tales estereotipos se consideran característicos de las mujeres y otra para indicar en qué medida éstos se consideran característicos de los hombres. Con la intención de favorecer una respuesta sobre la visión de los grupos de género considerados globalmente, y para evitar los efectos de la deseabilidad social y las normas sociales, que incitarían a responder de una forma no estereotípica, se evaluó la asignación de cada adjetivo para los hombres y las mujeres en general según la sociedad actual. Tras comprobar el mantenimiento de la asignación diferencial de los diferentes estereotipos, se analizó el efecto global de las características sociodemográficas sobre la consideración de cada estereotipo como más o menos característico de cada género. Finalmente, se señalaron los estereotipos de género actuales, es decir, aquellos que se continúan asignando diferencialmente y que se consideran característicos de cada género independientemente de aspectos como el sexo, estado civil u orientación política de los participantes. 


\section{Objetivos e hipótesis}

Los objetivos específicos de este estudio fueron, en primer lugar, comprobar la vigencia de los estereotipos de género tradicionales, y en segundo lugar, conocer si actualmente han surgido otros estereotipos de género. Un estereotipo de género se consideró vigente en nuestra sociedad si a) se asignó de forma diferencial a uno de los grupos de género, y b) no se encontró ningún efecto global de las variables de interés sobre la consideración de dicho estereotipo como más o menos característico de un grupo de género; es decir, el estereotipo se consideraba vigente si podíamos afirmar que éste se mantenía por igual en todas las personas independientemente de sus variables sociodemográficas, como el estado civil, la orientación política o la religiosidad.

Apoyándonos en los postulados de la teoría del rol social sobre el cambio en los estereotipos de género, se espera que la mayor presencia actual de las mujeres en ámbitos considerados tradicionalmente masculinos dé lugar a una modificación en las características asociadas principalmente con este grupo de género. No obstante, también se espera que la presencia de una jerarquía basada en el género en nuestra sociedad (como se refleja, por ejemplo, en la violencia contra las mujeres o en la propagación de información sexista) favorezca el mantenimiento de algunos estereotipos de género tradicionales. Además, en consonancia con estudios previos, se espera que algunas características sociodemográficas influyan sobre la consideración de un estereotipo como más o menos característico de los grupos de género. Concretamente, las hipótesis exploratorias planteadas para este estudio fueron las siguientes:

- Hipótesis 1: Algunos estereotipos de género tradicionales no se asignarían de forma diferencial a los grupos de género en la actualidad, considerándose no vigentes.

- Hipótesis 2: Algunas variables sociodemográficas, como el sexo o la orientación política, producirían un efecto global en la consideración de algunos estereotipos como más o menos característicos de hombres o mujeres. De esta manera, si la estimación de un estereotipo como característico de un género concreto se debiera al efecto de alguna variable sociodemográfica, dicho estereotipo no se consideraría vigente porque no podría afirmarse que su estimación se mantuviera para todas las personas independientemente de estas variables.

- Hipótesis 3: Aparecerían algunos estereotipos nuevos que se asignan diferencialmente a mujeres y hombres, y que se mantienen independientemente de las características sociodemográficas. Esto podría deberse bien a que la asignación tradicional de algunos estereotipos de género se invirtiera, de manera que en la actualidad se atribuyan al grupo de género contrario, o bien a que algunas de las características utilizadas para definir a mujeres y hombres que aún no han sido analizadas en estudios precedentes muestren una asignación diferencial significativa. Estos estereotipos serían nuevos y vigentes en la actualidad.
- Hipótesis 4: Aparte de los cambios señalados que se esperan observar en cuanto a la vigencia de los estereotipos de género, y teniendo en cuenta la presencia en nuestra sociedad de una jerarquía basada en el género, también esperaríamos que algunos de los estereotipos tradicionales se mantuvieran vigentes.

\section{Método}

\section{Participantes}

Un total de 164 personas colaboraron de forma voluntaria y anónima, recibiendo a cambio una puntuación extra para la nota de prácticas de una asignatura de su titulación. La distribución de la muestra en cuanto al sexo de los participantes fue equilibrada, de forma que 82 eran mujeres (50\%) y 82 eran hombres. La edad de los participantes oscilaba entre los 18 y 29 años $(M=20.68$ años; $D T=2.20)$. Todas las personas que participaron en esta investigación eran estudiantes de diferentes titulaciones de la Universidad de Jaén, correspondientes a las Facultades de Ciencias Sociales y Jurídicas $(35.3 \%)$ y Humanidades y Ciencias de la Educación (8.5\%), así como a las Escuelas de Ciencias de la Salud (11\%), Trabajo Social $(0.6 \%)$ y Politécnica Superior (4.9\%). Dadas las características de los objetivos de este estudio, ningún estudiante de la titulación de Psicología participó en él, pues su formación podría sesgar sus respuestas. Con respecto a otros datos sociodemográficos de interés, la muestra se distribuía de la siguiente forma: en relación a su orientación sexual, el 96.9\% indicaron una orientación heterosexual, el $2.5 \%$ homosexual y el $0.6 \%$ bisexual. En cuanto al estado civil, la mayoría de los participantes eran solteros (53.7\%) o solteros con pareja $(44.5 \%)$, mientras que el resto convivían con su pareja $(1.2 \%)$ o estaban casados $(0.6 \%)$. En relación a la orientación política, un $33.5 \%$ de los participantes manifestaron una orientación de izquierda, un $13.4 \%$ de centro y un $17.1 \%$ de derecha, mientras que un 36\% manifestó no mostrar ninguna orientación. Con respecto a la religión, un $54.9 \%$ eran creyentes, $23.8 \%$ agnósticos y $21.3 \%$ ateos. La selección de la muestra se realizó mediante un muestreo no probabilístico incidental y tipo bola de nieve para alcanzar una misma proporción de mujeres y hombres.

\section{Instrumentos}

Los instrumentos utilizados fueron los siguientes:

- Cuestionario Sociodemográfico: con este cuestionario se recogió información de los participantes con respecto a su edad, sexo (mujer/hombre), orientación sexual (heterosexual/homosexual/bisexual), estado civil (soltero-a/soltero-a con pareja/casado-a/separado-a/divorciado-a/viudoa/otro), titulación y curso (preguntas abiertas), orientación politica (izquierda/centro/derecha/ninguna), grado de orientación política (valorado con una escala tipo Likert de 7 puntos, desde "poco" hasta "mucho"), religiosidad (creyente/agnóstico-a/ateo-a), grado de religiosidad (valorado con 
una escala tipo Likert de 7 puntos, desde "poco" hasta "mucho"), y religión (pregunta abierta para indicar la religión que se profesa en su caso).

- Escala de Características Estereotípicas: la versión original de esta escala constaba de 258 adjetivos, cuyo proceso de construcción se explicará en el apartado de procedimiento. Estos adjetivos incluían estereotipos de género tradicionales y otras características que se suelen utilizar para describir a los grupos de género. A partir de la escala original se crearon dos versiones de manera que permitieran conocer por separado los estereotipos asociados con las mujeres (versión mujeres, Anexo 1) y con los hombres (versión hombres). Para ello, se realizaron las adaptaciones correspondientes de las instrucciones y de la terminación del adjetivo en función del género valorado. De esta forma, la escala completa contenía 516 adjetivos. Las instrucciones solicitaban que se señalara el grado de acuerdo con que cada adjetivo define a las mujeres (hombres) en general según la sociedad actual. Para ello, se utilizó un formato de respuesta tipo Likert de 7 puntos, donde 1 indicaba "totalmente en desacuerdo" y 7 indicaba "totalmente de acuerdo". Para cada versión, la frabilidad fue de $\alpha=.95$.

Las variables independientes de este estudio fueron las siguientes características sociodemográficas ${ }^{2}$ : el sexo, la edad, el estado civil, la orientación política y la religiosidad. Las variables dependientes fueron los adjetivos que formaban la escala completa.

\section{Procedimiento}

En cuanto al proceso de construcción de la escala de adjetivos, en primer lugar, se partió de la escala elaborada por Castillo-Mayén y Montes-Berges (2007) que consta de 242 adjetivos. Para la elaboración de esta escala se incluyeron estereotipos de género utilizados en estudios anteriores realizados tanto en España como en otros países (Garrido Lora, 2007; Langford y MacKinnon, 2000; Velasco Sacristán, 2003; Williams y Best, 1982), así como otros adjetivos empleados para describir comúnmente a los grupos de género. En el presente estudio se revisó dicha escala y se comparó este listado con los estereotipos incluidos en otras fuentes bibliográficas relevantes (ej., Eagly y Mladinic, 1989, 1993; López-Sáez, 2008; Montes-Berges, 2002; Morales y LópezSáez, 1993; Williams y Best, 1990), introduciendo aquellos que aún no figuraban. Esta revisión también dio lugar a la eliminación de algunos de los adjetivos incluidos en el listado original, debido a que habían causado problemas de comprensión en el estudio previo de las autoras (ej., labiosos/as), o por otras razones de contenido (ej., secos/as, miserables).

\footnotetext{
2 Consideramos que la orientación sexual también podría aportar información relevante en este estudio, pero la mayoría de participantes $(96.9 \%)$ ocupó sólo uno de los niveles, por lo que los análisis de esta variable no resultaban pertinentes.
}

Tras el consentimiento informado, se presentó a los participantes el cuestionario sociodemográfico descrito anteriormente, seguido de instrucciones generales para la correcta cumplimentación de la escala. A continuación, los participantes completaron las dos versiones de la escala de adjetivos, que se presentaron debidamente contrabalanceadas, de manera que en la mitad de las ocasiones se valoraba en primer lugar a las mujeres y a continuación a los hombres $(48.2 \%)$, y en la otra mitad se valoraba en primer lugar a los hombres y a continuación a las mujeres (51.8\%). La cumplimentación de las escalas se realizó en sesiones grupales.

\section{Diseño y análisis de datos}

El diseño utilizado a lo largo del estudio así como los análisis estadísticos realizados fueron los siguientes. En primer lugar, se utilizó un diseño intrasujetos para la cumplimentación de ambas versiones de la Escala de Características Estereotípicas (presentación contrabalanceada). Así, para conocer la asignación diferencial de cada característica a ambos géneros, se realizó un Análisis de Medidas Repetidas, siendo los 258 pares de adjetivos las variables dependientes. A continuación, tras eliminar los adjetivos que no se asignaron de manera diferencial a los grupos de género, se realizaron Análisis Multivariados de la Varianza con los 192 adjetivos restantes para cada versión de la escala y con cada variable independiente. Las variables independientes fueron sexo, con dos niveles (mujer/hombre), edad, con siete niveles $(18 / 19 / 20 / 21 / 22 / 23 / 24$ o más años), estado civil (solteroa/soltero-a con pareja), orientación política (izquier$\mathrm{da} /$ centro/derecha/ninguna), y religiosidad (creyente/agnóstico-a/ateo-a). A continuación, se realizaron contrastes entresujetos, y por último, se integraron los resultados obtenidos en el primer y último análisis, dando lugar al listado final de adjetivos.

\section{Resultados $^{3}$}

Para conocer la vigencia de los estereotipos de género se llevaron a cabo los siguientes análisis. En primer lugar, se realizó un Análisis de Medidas Repetidas para comprobar la asignación diferencial de los adjetivos a cada grupo de género. Los adjetivos que no mostraron diferencias en su asignación fueron un total de 66, y se eliminaron de la lista original (todas las $F_{\mathrm{S}}<3.86$, $p$ s $>$.051). De esta forma, cada versión de la escala quedó con un total de 192 adjetivos. Algunos de los estereotipos de género tradicionales que no se asignaron de manera diferencial a ningún género fueron autoeficaces, autónomos, dependientes, dóciles, complacientes, de bajo estatus, asertivos/as y dominantes.

\footnotetext{
${ }^{3}$ Por razones de espacio, no podemos incluir todos los resultados obtenidos en detalle debido al gran número de variables analizadas. Dichos resultados están disponibles contactando con la primera autora del artículo.
} 
En segundo lugar, se realizó un Análisis Multivariado de la Varianza con el objetivo de identificar el posible efecto global de las variables independientes sobre los adjetivos que habían mostrado una asignación diferencial a mujeres y hombres. Los resultados de los contrastes multivariados sólo fueron significativos al considerar el efecto del estado civil en los adjetivos utilizados para definir a las mujeres, Traza de Pillai $=1.00, F(1,58)=12241.87, p=.007, \eta_{p}^{2}=1.00$. Considerando los resultados de las pruebas de los efectos inter-sujetos, presentamos, a modo de ejemplo y para resumir los resultados, algunos de los adjetivos en los que se encontró un efecto de estas variables ${ }^{4}$. Dado que no se realizó el ajuste para comparaciones múltiples, sus implicaciones serán consideradas en la discusión.

En relación a la variable sexo, ésta produjo un efecto global en 35 adjetivos cuando se utilizaron para definir a las mujeres en general. Sin embargo, cuando los adjetivos se utilizaron para definir a los hombres, se encontró un efecto global de la variable sexo en 13 adjetivos. Considerando los resultados de esta variable en ambas versiones de la escala, se observa que el efecto global del sexo se encontró en más

${ }^{4}$ El motivo por el cual los grados de libertad del denominador para el estadístico $F$ sugieren la presencia de valores perdidos se debe a que el programa estadístico utilizado para el análisis de datos (SPSS), al igual que la mayoría de programas para tal uso, utiliza el análisis de casos completos (list wise deletion) por defecto. De esta forma, todos los casos que presentan uno o más datos vacíos se eliminan antes de ejecutar el Análisis Multivariado de la Varianza, asumiendo que los valores perdidos se produjeron de manera aleatoria. mente las diferencias entre mujeres y hombres al describir a las mujeres (Figura 1) y al describir a los hombres. (Figura 2).

Con respecto a la variable edad, se tuvieron en cuenta un total de 7 niveles, agrupándose en un único nivel a los participantes con edades iguales o superiores a los 24 años. Se realizó esta agrupación para equiparar el tamaño de los niveles de esta variable, ya que su frecuencia a partir de los 24 años oscilaba entre uno y cuatro participantes. La variable edad produjo un efecto global en 14 adjetivos cuando se utilizaron para definir a las mujeres, como en delicadas, $F(6,54)$ $=3.96, p=.002, \eta^{2}{ }_{p}=.306, \mathrm{y}$ bumildes, $F(6,54)=3.41, p=$ $.006, \eta_{p}^{2}=.275$. Al utilizar los adjetivos para definir a los hombres, el efecto global de la variable edad se encontró en 10 adjetivos, como en sexualmente activos, $F(6,54)=2.54, p=$ $.03, \eta_{p}{ }_{p}=.220$, e infieles, $F(6,54)=4.10, p=.002, \eta_{p}{ }_{p}=.313$.

En relación al estado civil, únicamente se tuvieron en cuenta los niveles "soltero/a" y "soltero/a con pareja", dado que el resto de niveles indicados por los participantes ("conviviendo con su pareja" y "casado-a") estaba formado por un total de tres personas. El efecto global del estado civil se obtuvo en 10 adjetivos cuando éstos se utilizaron para definir a las mujeres, como en honradas, $F(1,58)=10.60, p=$ $.002, \eta_{p}^{2}=.155$, y educadas, $F(1,58)=6.54, p=.036, \eta_{p}{ }_{p}=$ .074 , y en 13 adjetivos cuando se utilizaron para definir a los hombres, como en imprevisibles, $F(1,58)=4.63, p=.036, \eta^{2}$ $=.074, \mathrm{y}$ sensatos, $F(1,58)=5.03, p=.029, \eta_{p}{ }^{2}=.08 \mathrm{El}$ efecto sobre los adjetivos "románticos/as", "peligrosos/as" y "agresivos/as" se encontró en ambas versiones.

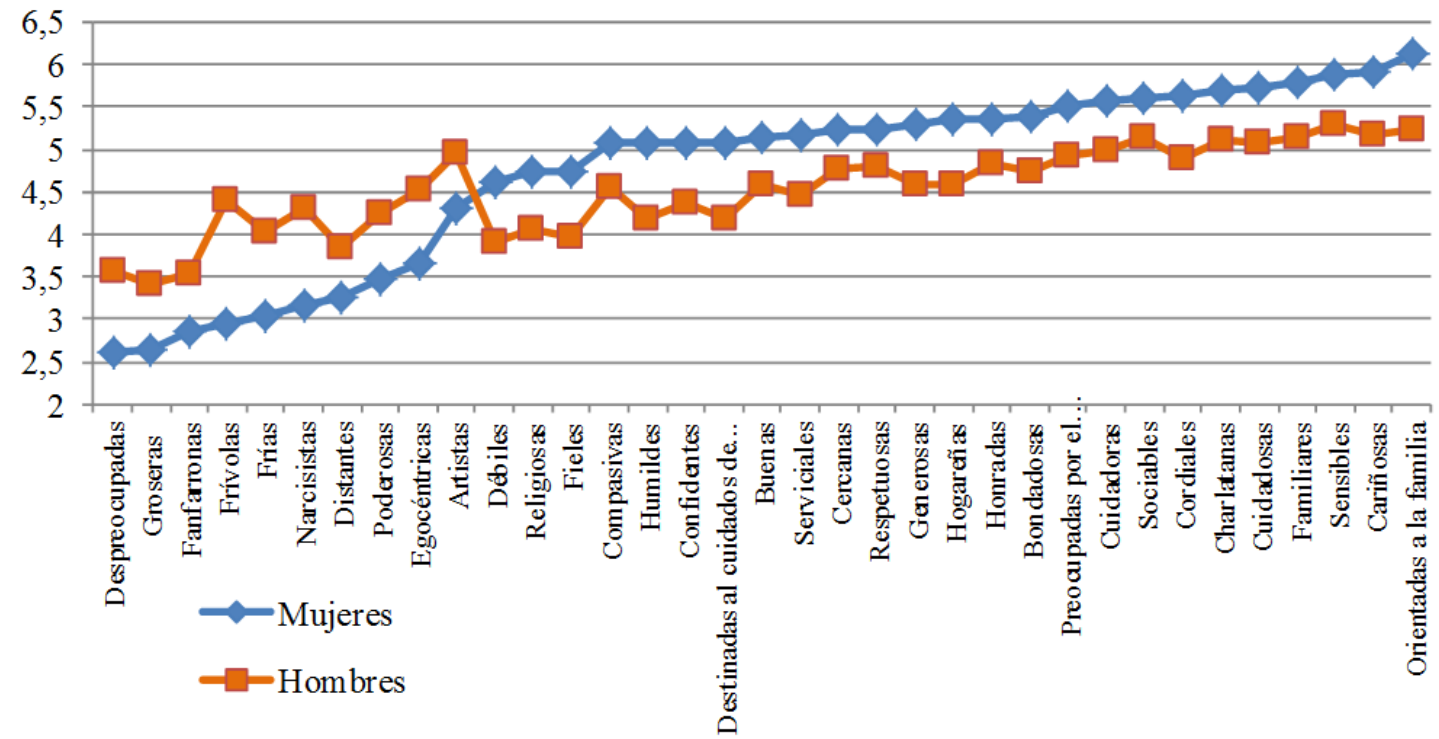

Figura 1. Representación gráfica de las medias de los adjetivos al definir a las mujeres según el sexo de los participantes (adjetivos ordenados de menor a mayor en función del valor de su media en esta versión de la escala). 


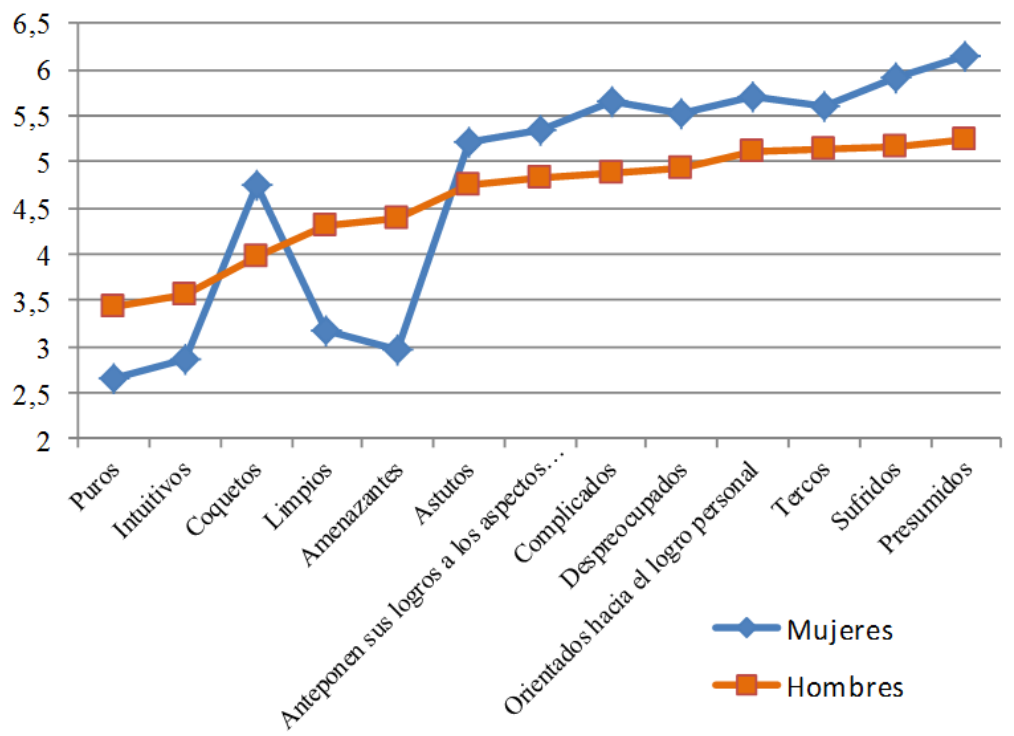

Figura 2. Representación gráfica de las medias de los adjetivos al definir a los hombres según el sexo de los participantes (adjetivos ordenados de menor a mayor en función del valor de su media en esta versión de la escala).

Con respecto a la orientación política, el efecto global de esta variable cuando los adjetivos se utilizaron para definir a las mujeres se encontró en 18 adjetivos, como en presumidas, $F(3,57)=3.14, p=.032, \eta_{p}^{2}=.142$, y en familiares, $F(3,57)$ $=7.74, p<.001, \eta_{p}^{2}=.289$. Sin embargo, el efecto global de la orientación política cuando los adjetivos se utilizaron para definir a los hombres, se obtuvo en 3 adjetivos, dos de los cuales fueron atentos, $F(3,57)=3.59, p=.019, \eta_{p}^{2}=.159, \mathrm{y}$ oprimidos, $F(3,57)=3.77, p=.015, \eta_{p}^{2}=.166$.

Al considerar los resultados de esta variable en ambas versiones de la escala, se observa que la orientación política produjo un efecto global sobre más adjetivos cuando estos se emplearon para definir a las mujeres (18) que cuando se emplearon para definir a los hombres (3). Ninguno de los adjetivos coincidió en las dos versiones.

En relación a la religiosidad, esta variable produjo un efecto global en 22 adjetivos cuando se utilizaron para definir a las mujeres, como en frivolas, $F(2,58)=4.68, p=.013, \eta_{p}^{2}=$ .139, y bondadosas, $F(2,58)=7.86, p=.001, \eta^{2}{ }_{p}=.213$. Cuando los adjetivos se utilizaron para definir a los hombres, se obtuvo un efecto global de la variable religiosidad en 7 adjetivos, como en puros, $F(2,58)=5.06, p=.009, \eta_{p}{ }^{2}=$ .149 , e imprudentes, $F(2,58)=3.85, p=.027, \eta_{p}^{2}=.117$.

Nuevamente, los resultados en relación a esta variable revelaron un efecto global en más adjetivos cuando estos se utilizaron para definir a las mujeres (22) que cuando se utili- zaron para definir a los hombres (7). Ninguno de los adjetivos se encontró en ambas versiones de la escala.

En relación a la potencia estadística de las pruebas utilizadas, se realizó un cálculo a posteriori de la diferencia mínima detectable basado en una prueba $t$ de los dos subgrupos muestrales más pequeños (orientación política centro, $13.4 \%, \mathrm{n}=22$, y orientación política derecha, $17.1 \%, \mathrm{n}=$ 28). Dicho cálculo mostró una potencia del $93 \%$ para detectar una diferencia de 1 punto (asumiendo una desviación típica de 1), con una tasa de error tipo I de .05.

Una vez realizados todos los análisis, el adjetivo era excluido del listado final si el efecto se había encontrado en alguna de las dos versiones de la escala o en ambas. Por último, se integraron los resultados del Análisis de Medidas Repetidas y del Análisis Multivariado de la Varianza para recoger los estereotipos de género actuales. De esta forma, se identificaron un total de 93 adjetivos que se habían asignado diferencialmente a los grupos de género y sobre los que ninguna variable independiente había ejercido un efecto global. En la Tabla 1, se muestra el listado de los adjetivos que se asignaron diferencialmente a las mujeres, formado por un total de 58. Se indica la media y desviación típica del adjetivo en cada versión de la escala. En la Tabla 2, se muestran las medias y desviaciones típicas en cada versión de la escala de los adjetivos que fueron asignados diferencialmente a los hombres. Estos adjetivos formaron un total de 35. En ambas tablas, la columna "número" indica el orden de aparición del adjetivo en la escala. 
Tabla 1. Media y desviación típica en cada versión de la escala de las características estereotípicas asignadas a las mujeres.

\begin{tabular}{|c|c|c|c|c|c|c|c|c|}
\hline \multirow[b]{2}{*}{$\mathrm{N}^{\circ}$} & \multirow[b]{2}{*}{ ADJETIVO } & \multicolumn{2}{|c|}{ MUJERES } & \multicolumn{2}{|c|}{ HOMBRES } & \multirow[b]{2}{*}{$F(1,163)$} & \multirow[b]{2}{*}{$p$} & \multirow[b]{2}{*}{$\eta_{p}^{2}$} \\
\hline & & $M$ & $D T$ & $M$ & $D T$ & & & \\
\hline 5 & Con alta habilidad verbal & 5.16 & 1.04 & 4.23 & 1.17 & 62.35 & $<.001$ & .278 \\
\hline 7 & $\begin{array}{l}\text { Piensan que las relaciones } \\
\text { humanas son importantes }\end{array}$ & 5.65 & 1.18 & 4.28 & 1.25 & 106.98 & $<.001$ & .396 \\
\hline 8 & Exigentes & 5.33 & 1.06 & 4.98 & 1.26 & 7.58 & .007 & .045 \\
\hline 10 & Habladoras & 5.54 & 1.14 & 4.12 & 1.37 & 99.93 & $<.001$ & .380 \\
\hline 12 & Volubles & 4.36 & 1.15 & 3.84 & 0.92 & 18.44 & $<.001$ & .105 \\
\hline 25 & Afectivas & 5.34 & 0.90 & 3.98 & 1.15 & 124.29 & $<.001$ & .437 \\
\hline 26 & Emocionales & 5.70 & 0.92 & 3.64 & 1.28 & 240.13 & $<.001$ & .603 \\
\hline 33 & Supersticiosas & 4.55 & 1.17 & 3.12 & 1.34 & 103.90 & $<.001$ & .389 \\
\hline 35 & Vengativas & 4.82 & 1.38 & 4.35 & 1.32 & 9.97 & .002 & .058 \\
\hline 36 & Comprensivas & 5.16 & 1.09 & 4.08 & 1.16 & 64.96 & $<.001$ & .285 \\
\hline 39 & Sentimentales & 5.66 & 0.97 & 3.71 & 1.20 & 239.39 & $<.001$ & .601 \\
\hline 46 & Calculadoras & 4.73 & 1.53 & 4.26 & 1.29 & 7.34 & .008 & .047 \\
\hline 48 & Maduras & 5.39 & 1.09 & 3.80 & 1.34 & 117.59 & $<.001$ & .424 \\
\hline 49 & Progresistas & 4.88 & 1.15 & 4.28 & 1.02 & 23.28 & $<.001$ & .126 \\
\hline 50 & Depresivas & 4.30 & 1.35 & 3.20 & 1.20 & 55.47 & $<.001$ & .260 \\
\hline 56 & Caprichosas & 5.09 & 1.39 & 4.03 & 1.34 & 49.34 & $<.001$ & .238 \\
\hline 65 & Nerviosas & 4.48 & 1.22 & 4.17 & 1.22 & 5.63 & .019 & .034 \\
\hline 74 & Quisquillosas & 4.71 & 1.24 & 4.05 & 1.38 & 23.93 & $<.001$ & .131 \\
\hline 78 & Saludables & 5.01 & 1.12 & 4.48 & 1.33 & 20.02 & $<.001$ & .110 \\
\hline 85 & Encantadoras & 5.22 & 1.08 & 4.16 & 1.01 & 95.73 & $<.001$ & .376 \\
\hline 87 & Sumisas & 3.89 & 1.47 & 3.27 & 1.17 & 17.75 & $<.001$ & .101 \\
\hline 92 & Pesimistas & 4.12 & 1.25 & 3.57 & 1.17 & 16.34 & $<.001$ & .091 \\
\hline 95 & Obsesivas & 4.59 & 1.10 & 4.09 & 1.08 & 23.87 & $<.001$ & .131 \\
\hline 100 & Trabajadoras & 5.31 & 1.05 & 5.10 & 1.10 & 4.35 & .039 & .026 \\
\hline 105 & Actúan con franqueza & 4.39 & 1.03 & 4.12 & 1.07 & 5.68 & .018 & .037 \\
\hline 109 & Enrevesadas & 4.81 & 1.22 & 3.90 & 1.23 & 41.79 & $<.001$ & .206 \\
\hline 116 & Modernas & 5.34 & 0.96 & 4.83 & 1.16 & 25.05 & $<.001$ & .135 \\
\hline 123 & Inteligentes & 5.31 & 1.07 & 4.88 & 1.01 & 15.09 & $<.001$ & .085 \\
\hline 129 & Respetables & 4.91 & 1.23 & 4.64 & 0.93 & 4.75 & .031 & .029 \\
\hline 130 & Nobles & 4.70 & 1.03 & 4.44 & 1.10 & 7.03 & .009 & .042 \\
\hline 133 & Maniáticas & 4.69 & 1.41 & 3.84 & 1.25 & 45.29 & $<.001$ & .218 \\
\hline 139 & Con buen gusto & 5.26 & 1.03 & 4.06 & 1.18 & 109.75 & $<.001$ & .405 \\
\hline 147 & Amables & 5.15 & 0.94 & 4.53 & 0.99 & 41.11 & $<.001$ & .207 \\
\hline 149 & Instructivas & 4.49 & 0.93 & 4.25 & 0.89 & 6.10 & .015 & .037 \\
\hline 155 & Amigables & 5.16 & 0.90 & 4.91 & 0.89 & 8.36 & .004 & .049 \\
\hline 158 & Dulces & 5.29 & 0.89 & 3.81 & 1.19 & 166.19 & $<.001$ & .508 \\
\hline 180 & Pacíficas & 4.90 & 1.05 & 4.13 & 1.04 & 44.59 & $<.001$ & .221 \\
\hline 182 & Leales & 4.80 & 1.10 & 4.16 & 1.19 & 27.50 & $<.001$ & .147 \\
\hline 185 & Minuciosas & 4.58 & 1.11 & 3.79 & 1.21 & 33.04 & $<.001$ & .170 \\
\hline 187 & Desquiciadas & 3.72 & 1.52 & 3.40 & 1.14 & 5.03 & .026 & .031 \\
\hline 190 & Afectuosas & 5.03 & 0.91 & 4.13 & 0.98 & 63.12 & $<.001$ & .288 \\
\hline 195 & Atractivas & 5.45 & 1.04 & 4.80 & 1.13 & 43.10 & $<.001$ & .210 \\
\hline 209 & Ingenuas & 4.43 & 1.20 & 3.94 & 1.19 & 14.80 & $<.001$ & .086 \\
\hline 211 & Lloronas & 5.10 & 1.33 & 2.75 & 1.40 & 212.81 & $<.001$ & .568 \\
\hline 220 & Curiosas & 5.34 & 1.06 & 4.37 & 1.20 & 52.57 & $<.001$ & .246 \\
\hline 221 & Vanidosas & 4.54 & 1.14 & 4.30 & 0.98 & 5.56 & .020 & .034 \\
\hline 222 & Protegidas & 4.68 & 1.07 & 3.68 & 1.22 & 65.05 & $<.001$ & .292 \\
\hline 224 & Cultas & 4.94 & 1.08 & 4.33 & 1.06 & 29.89 & $<.001$ & .163 \\
\hline 225 & Solidarias & 5.06 & 1.06 & 4.13 & 1.06 & 77.88 & $<.001$ & .326 \\
\hline 228 & Protectoras & 5.16 & 1.13 & 4.80 & 1.06 & 8.57 & .004 & .050 \\
\hline 235 & Víctimas & 4.08 & 1.44 & 3.15 & 1.21 & 40.04 & $<.001$ & .203 \\
\hline 240 & Racionales & 4.48 & 1.16 & 4.13 & 0.99 & 8.82 & .003 & .052 \\
\hline 248 & Sutiles & 4.60 & 1.03 & 3.94 & 1.08 & 31.10 & $<.001$ & .163 \\
\hline 249 & Vulnerables & 4.37 & 1.23 & 3.59 & 1.15 & 33.08 & $<.001$ & .172 \\
\hline 250 & Inseguras & 4.37 & 1.40 & 3.76 & 1.25 & 17.06 & $<.001$ & .095 \\
\hline 252 & Agradables & 5.11 & 0.88 & 4.72 & 0.97 & 14.60 & $<.001$ & .084 \\
\hline 256 & Moralistas & 4.30 & 0.99 & 3.83 & 0.91 & 23.68 & $<.001$ & .129 \\
\hline 258 & Incondicionales & 4.24 & 1.03 & 3.93 & 1.04 & 7.04 & .009 & .041 \\
\hline
\end{tabular}


Tabla 2. Media y desviación típica en cada versión de la escala de las características estereotípicas asignadas a los hombres.

\begin{tabular}{|c|c|c|c|c|c|c|c|c|}
\hline \multirow[b]{2}{*}{$\mathrm{N}^{\circ}$} & \multirow[b]{2}{*}{ ADJETIVO } & \multicolumn{2}{|c|}{ MUJERES } & \multicolumn{2}{|c|}{ HOMBRES } & \multirow[b]{2}{*}{$F(1,163)$} & \multirow[b]{2}{*}{$p$} & \multirow[b]{2}{*}{$\eta_{p}^{2}$} \\
\hline & & $M$ & DT & $M$ & DT & & & \\
\hline 17 & Con alta habilidad en matemáticas & 3.98 & 1.13 & 4.56 & 1.26 & 14.28 & $<.001$ & .081 \\
\hline 18 & Egoístas & 3.59 & 1.21 & 4.55 & 1.30 & 55.45 & $<.001$ & .255 \\
\hline 21 & Perezosos & 3.04 & 1.26 & 4.51 & 1.44 & 101.51 & $<.001$ & .385 \\
\hline 27 & Valientes & 4.39 & 1.40 & 5.22 & 1.07 & 30.36 & $<.001$ & .158 \\
\hline 40 & Arriesgados & 4.05 & 1.18 & 5.00 & 0.99 & 54.23 & $<.001$ & .252 \\
\hline 41 & Destinados al placer sexual & 3.97 & 1.60 & 4.75 & 1.69 & 23.82 & $<.001$ & .128 \\
\hline 57 & Poco emocionales & 2.60 & 1.21 & 4.42 & 1.37 & 163.23 & $<.001$ & .507 \\
\hline 58 & Emprendedores & 4.53 & 1.11 & 4.88 & 1.05 & 7.26 & .008 & .043 \\
\hline 59 & Solitarios & 3.38 & 1.30 & 3.99 & 1.18 & 19.55 & $<.001$ & .110 \\
\hline 60 & Impulsivos & 4.32 & 1.31 & 4.85 & 1.16 & 13.49 & $<.001$ & .078 \\
\hline 62 & Maleducados & 2.92 & 1.17 & 3.96 & 1.36 & 68.28 & $<.001$ & .297 \\
\hline 63 & Líderes & 3.88 & 1.12 & 5.11 & 1.15 & 84.75 & $<.001$ & .349 \\
\hline 68 & Viciosos & 3.76 & 1.43 & 4.85 & 1.29 & 62.02 & $<.001$ & .278 \\
\hline 70 & Incompletos & 3.46 & 1.47 & 3.79 & 1.35 & 5.27 & .023 & .032 \\
\hline 71 & Conflictivos & 3.47 & 1.40 & 4.55 & 1.32 & 60.15 & $<.001$ & .276 \\
\hline 72 & Rebeldes & 3.72 & 1.42 & 4.48 & 1.25 & 26.60 & $<.001$ & .143 \\
\hline 79 & Fuertes emocionalmente & 4.20 & 1.60 & 4.72 & 1.50 & 7.47 & .007 & .044 \\
\hline 102 & Naturales & 4.33 & 1.27 & 4.66 & 1.07 & 5.21 & .024 & .033 \\
\hline 127 & Insensibles & 2.73 & 1.09 & 4.09 & 1.29 & 117.95 & $<.001$ & .432 \\
\hline 132 & Promiscuos & 3.99 & 1.22 & 4.51 & 1.21 & 17.36 & $<.001$ & .099 \\
\hline 135 & Defectuosos & 3.17 & 1.40 & 3.86 & 1.42 & 29.30 & $<.001$ & .156 \\
\hline 145 & Graciosos & 4.21 & 1.15 & 5.03 & 1.08 & 45.54 & $<.001$ & .219 \\
\hline 177 & Fuertes físicamente & 3.11 & 1.19 & 5.46 & 1.22 & 272.56 & $<.001$ & .630 \\
\hline 183 & Amantes del peligro & 3.29 & 1.22 & 4.79 & 1.09 & 131.05 & $<.001$ & .449 \\
\hline 184 & Duros & 3.48 & 1.33 & 4.84 & 1.24 & 79.67 & $<.001$ & .332 \\
\hline 215 & Juerguistas & 4.44 & 1.16 & 5.48 & 1.09 & 77.21 & $<.001$ & .326 \\
\hline 216 & Firmes & 4.35 & 1.11 & 4.66 & 0.98 & 6.94 & .009 & .041 \\
\hline 223 & Individualistas & 4.08 & 1.08 & 4.47 & 1.14 & 14.25 & $<.001$ & .082 \\
\hline 230 & Autoritarios & 4.38 & 1.22 & 4.78 & 1.08 & 12.18 & .001 & .070 \\
\hline 233 & Pasivos & 3.43 & 1.24 & 3.85 & 1.21 & 10.40 & .002 & .061 \\
\hline 237 & Violentos & 2.81 & 1.25 & 3.89 & 1.32 & 68.66 & $<.001$ & .303 \\
\hline 239 & Sucios & 2.43 & 1.24 & 3.55 & 1.42 & 60.81 & $<.001$ & .277 \\
\hline 241 & Inexpresivos & 2.88 & 1.31 & 4.00 & 1.16 & 78.03 & $<.001$ & .332 \\
\hline 253 & De personalidad fuerte & 4.47 & 1.24 & 4.92 & 1.02 & 11.95 & .001 & .071 \\
\hline 254 & Atléticos & 3.53 & 1.19 & 5.18 & 1.15 & 152.77 & $<.001$ & .488 \\
\hline
\end{tabular}

Para destacar los adjetivos más relevantes asignados a cada género diferencialmente, a continuación se representan de manera gráfica las 10 características estereotípicas asignadas a las mujeres con una mayor diferencia en su media con respecto a su asignación a los hombres (Figura 3) y las 10 características estereotípicas asignadas a los hombres que mostraron una mayor diferencia en su media con respecto a su asignación a las mujeres (Figura 4).

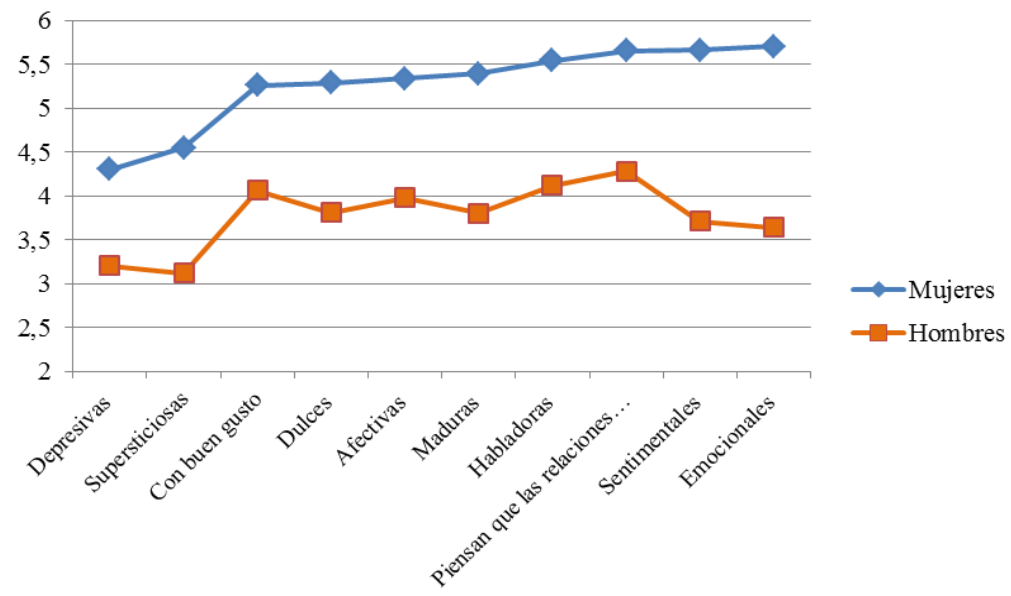

Figura 3. Representación gráfica de las características estereotípicas más relevantes asignadas diferencialmente a las mujeres en comparación con su asignación a los hombres. 


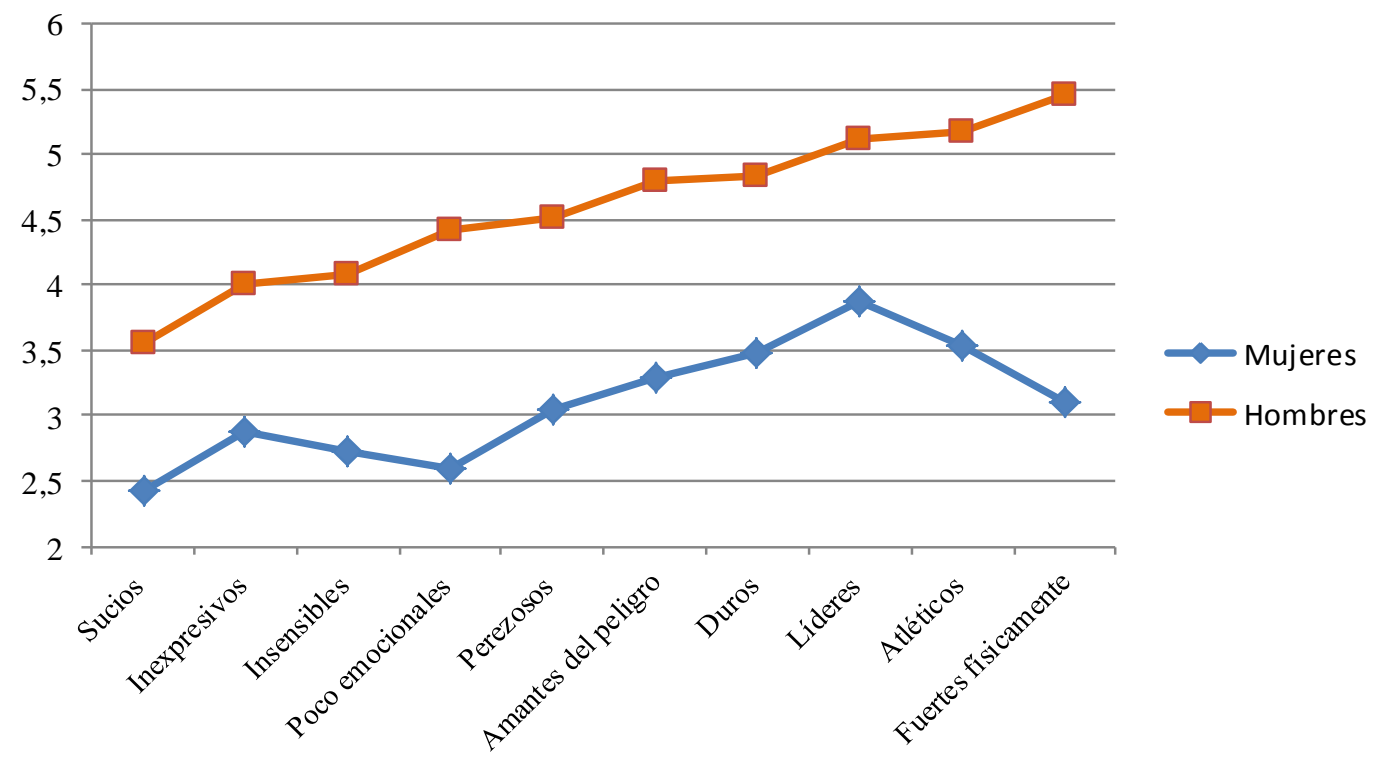

Figura 4. Representación gráfica de las características estereotípicas más relevantes asignadas diferencialmente a los hombres en comparación con su asignación a las mujeres.

\section{Discusión}

Con este estudio se pretendía conocer la vigencia actual de los estereotipos de género y señalar el surgimiento de otros estereotipos nuevos. Para ello, se ha comprobado la asignación diferencial de las características estereotípicas a cada género y el posible efecto global de las variables sociodemográficas de interés en su consideración como más o menos característicos de cada grupo. En general, los resultados obtenidos permiten apoyar las hipótesis planteadas. En relación con la primera hipótesis, en la que se esperaba encontrar que algunos estereotipos de género tradicionales no se asignaran diferencialmente a ningún género en la actualidad, se puede observar que efectivamente estereotipos femeninos tradicionales como dependientes, dóciles, complacientes o destinadas a la reproducción, y estereotipos masculinos tradicionales como autoeficaces, competentes, triunfadores o activos, no fueron asignados diferencialmente a hombres ni a mujeres en este estudio. Así, podemos afirmar que estos estereotipos han dejado de ser vigentes en la actualidad. Estos resultados están en consonancia con las predicciones de la teoría del rol social (Eagly, 1987; Eagly et al., 2000; Eagly et al., 2004), según la cual los cambios que se producen en los roles sociales generan un cambio en los estereotipos de género. De acuerdo con esta teoría, la división del trabajo es una de las causas de las diferencias conductuales observadas en hombres y mujeres, lo que contribuye en la asignación de características estereotípicas a cada grupo de género. De esta forma, teniendo en cuenta la mayor presencia de las mujeres en la actualidad en ámbitos considerados tradicionalmente masculinos, se espe- raría un cambio en tales características, tal y como se pone de manifiesto en este estudio.

La segunda hipótesis planteada, en la que se esperaba encontrar el efecto global de las variables sociodemográficas sobre algunos estereotipos, también se ve apoyada por los resultados. Las variables independientes que produjeron un efecto global sobre un mayor número de adjetivos fueron, en este orden, el sexo y la religiosidad, seguidas de la edad, estado civil y orientación política. De estos resultados se desprende que, en función de ciertas variables sociodemográficas como las tenidas en cuenta en este estudio, se encuentran diferencias en la consideración de los estereotipos como más o menos característicos de los grupos de género. Además, se observa que a excepción del estado civil, el efecto de estas variables se produjo en mayor medida sobre los adjetivos utilizados para definir a las mujeres en general. Es importante también señalar que más de una variable coincidió en mostrar este efecto sobre varios estereotipos femeninos tradicionales cuando se preguntaba por su adecuación para definir a las mujeres. Por ejemplo, se encontraron diferencias significativas en el adjetivo orientadas a la familia al considerar las variables sexo, edad, orientación política y religiosidad, en los adjetivos cercanas y débiles al considerar las variables sexo y edad, y en sensibles al considerar el sexo y la religiosidad. El hecho de que las características sociodemográficas den lugar a más diferencias cuando los adjetivos se utilizan para definir a las mujeres que cuando se utilizan para definir a los hombres, subraya la conveniencia de tener en cuenta tales variables para un análisis más profundo de la dinámica de los estereotipos. No obstante, a pesar de la utilidad de estos resultados, se debe señalar que si se hubiera 
realizado una corrección de Bonferroni para comparaciones múltiples, sólo los adjetivos frívolas y bumildes en el análisis de la variable sexo, y familiares en el análisis de la orientación política (todos referidos a la versión de la escala para describir a las mujeres) hubieran sido significativos $(p<.001)$. Sin embargo, consideramos que esta corrección puede ser excesivamente estricta, y por lo tanto se ha expuesto la interpretación de todas las pruebas realizadas para la consideración de sus posibles implicaciones en este ámbito de estudio.

Asimismo, la mayor variabilidad encontrada en los estereotipos utilizados para definir a las mujeres se relaciona con las conclusiones obtenidas en otros estudios, donde se ha observado una mayor dinámica en el estereotipo femenino (ej., Diekman y Eagly, 2000; Diekman et al., 2005; GarcíaRetamero et al., 2009; López-Sáez et al., 2008; López-Zafra et al., 2008). La teoría del rol social también permite explicar este resultado, puesto que son los roles sociales femeninos los que han experimentado un mayor cambio en las últimas décadas. Así, en la actualidad, los roles tradicionales asociados con las mujeres (ej., cuidar a los demás, realizar las tareas domésticas) continúan vinculándose con ellas en mayor medida que con los hombres, mientras que los roles tradicionales asociados a los hombres (ej., trabajar fuera del hogar, ocupar puestos directivos) se siguen vinculando con ellos pero también, cada vez más, con las mujeres. De esta manera, dado que los roles sociales que ocupan los hombres han sufrido pocos cambios en los últimos años (SánchezHerrero Arbide, Sánchez-López y Dresch, 2009), es comprensible que se encuentre una mayor estabilidad en los estereotipos masculinos, y que por tanto exista un mayor acuerdo en éstos independientemente de las características sociodemográficas de las personas que los perciben. Sin embargo, los roles sociales ocupados por las mujeres, al haberse diversificado, generan un mayor cambio en los estereotipos femeninos, explicándose que su asignación se vea más afectada por tales características.

En relación a la tercera hipótesis planteada, también se ha visto apoyada al encontrarse estereotipos de género que pueden considerarse nuevos. Concretamente, se observa que actualmente se asigna a las mujeres estereotipos masculinos tradicionales como inteligentes y racionales, y a los hombres estereotipos femeninos tradicionales como incompletos y pasivos. Es decir, en la actualidad, algunos estereotipos tradicionales se asignan al género contrario, resultado que también se había encontrado en estudios previos (ej., Moya y Pérez, 1990). Además, se ha observado que algunas de las características utilizadas comúnmente para describir a las mujeres que no suelen aparecer en la literatura, se asignan diferencialmente a este grupo (ej., habladoras, calculadoras, nerviosas, trabajadoras, ingenuas o solidarias), lo que aporta un mayor conocimiento sobre los estereotipos de género actuales.

No obstante, a pesar de los cambios descritos hasta ahora sobre la dinámica de los estereotipos, en este estudio también se ha evidenciado que numerosos estereotipos de género tradicionales se mantienen en la actualidad (Hipótesis 4). Concretamente, características como egoístas, fuertes física- mente, insensibles o valientes continúan considerándose como más propias de los hombres, y características como sumisas, dulces, emocionales o comprensivas de las mujeres. Este resultado es esperable en la medida en que la existencia de un sistema jerárquico basado en el género forma parte de nuestra realidad, como se observa, por ejemplo, en la persistencia de la violencia contra las mujeres, la discriminación salarial, la menor presencia de mujeres en puestos de liderazgo, o en la perpetuación de los estereotipos y los roles de género en una publicidad marcadamente sexista. Además, no podemos olvidar que el mantenimiento de dicha estructura jerárquica conlleva consecuencias negativas también para los hombres, implicando expectativas de conducta y roles específicos también para este género, como puede desprenderse, por ejemplo, de la Figura 4. Así, como señalábamos al iniciar este trabajo, es importante tener en cuenta que el mantenimiento de los estereotipos de género repercute negativamente tanto en mujeres como en hombres, aunque sus consecuencias son mucho más negativas para el primer grupo.

Una aportación añadida de nuestro estudio es la elaboración de instrumentos que se podrían utilizar en investigaciones futuras y que permitirían realizar contribuciones relevantes en otros ámbitos de estudio. Por ejemplo, el listado completo de adjetivos podría utilizarse para conocer los estereotipos que se asocian a grupos sociales que se pueden categorizar basándonos en el género y en otra categoría, como por ejemplo la inmigración (ej., mujeres inmigrantes-mujeres no inmigrantes), la discapacidad (ej., mujeres con discapacidad-mujeres sin discapacidad), el liderazgo (ej., mujeres líderes-hombres líderes), la homosexualidad (ej., mujer homosexual-hombre homosexual), etc. En este sentido, el listado completo de adjetivos se ha aplicado recientemente para conocer los estereotipos con los que se describe a las mujeres en situación de maltrato (Montilla, Aranda y Montes-Berges, 2010), y para comparar los estereotipos asignados a hombres y mujeres cuando se desempeñan roles sociales (in)congruentes con el grupo de género, concretamente en el sector sanitario de la enfermería (Montes-Berges, 2010).

Respecto a las limitaciones de este estudio, estas se refieren principalmente a la generalización de los resultados obtenidos. Así, estos resultados no se pueden considerar como una imagen completa de la dinámica de los estereotipos en nuestra sociedad teniendo en cuenta la muestra utilizada, aunque sí permiten afirmar que estas variaciones en la asignación de estereotipos de género se están produciendo, al menos, en personas jóvenes universitarias. Por lo tanto, sería conveniente que en investigaciones futuras este estudio se repitiera con muestras más heterogéneas en cuanto a la edad, la ocupación, el nivel de estudios, el lugar de origen, el estado civil y la orientación sexual, entre otras variables de interés, manteniendo equilibrada la distribución en cuanto al sexo de los participantes. En esta línea, la replicación de este estudio en otros países permitiría además comparar la dinámica de los estereotipos de género y los fenómenos subyacentes en diferentes contextos culturales. 


\section{Conclusiones}

A partir de los resultados obtenidos en este estudio, se pueden establecer una serie de conclusiones sobre la dinámica de los estereotipos de género en nuestra sociedad en el momento actual, y por ende, sobre la discriminación de género. En este estudio se han identificado los estereotipos de género actuales, observándose tanto algunos cambios importantes en la asignación diferencial de características estereotípicas a los grupos de género como el mantenimiento de estereotipos tradicionales para describir a mujeres y a hombres. Además, la consideración del efecto global de las variables sociodemográficas ha permitido establecer los estereotipos que se perciben como característicos de cada género en la misma medida para todas las personas, independientemente de aspectos como el sexo, la edad, el estado civil, la orientación política o la religiosidad de los participantes. En general, los resultados que se derivan de esta investigación son acordes con la teoría del rol social, según la cual se espera un cambio en los estereotipos de género como consecuencia de los cambios que se producen en la ocupación de los roles sociales.

Concretamente, en primer lugar se ha señalado que algunos estereotipos de género tradicionales dejan de ser vigentes en la actualidad. Esta conclusión se obtiene, por un lado, tras comprobar que algunos estereotipos no se asignaron diferencialmente a ningún género (ej., dóciles, complacientes, independientes, activos). Por otro lado, se ha observado que las características sociodemográficas ejercían un efecto global sobre la consideración de algunos estereotipos como más o menos característicos de uno u otro género (ej., carinosas, sociables, despreocupados, poderosos). En segundo lugar, se han señalado algunos estereotipos de género que se consideran nuevos, bien porque tradicionalmente se asociaban con el grupo de género contrario (ej., se ha asignado el adjetivo pasivos a los hombres e inteligentes a las mujeres), o bien porque no se habían analizado en estudios precedentes (ej., solidarias y solitarios).

Es importante señalar que el efecto global observado de las características sociodemográficas sobre los estereotipos de género sugiere que las personas, en función de aspectos como el sexo, la religiosidad o el estado civil, mantienen representaciones distintas sobre los hombres y las mujeres. Además, estas características produjeron un efecto global sobre un mayor número de adjetivos cuando estos se utilizaban para definir a las mujeres, lo que podría deberse a la ma-

\section{Referencias}

Barberá, E. (1998). Psicología del género. Barcelona: Ariel Psicología.

Bem, S. L. (1974). The measurement of psychological androgyny. Journal of Consulting and Clinical Psychology, 42(2), 155-162.

Burgess, D. y Borgida, E. (1999). Who women are, who women should be: Descriptive and prescriptive gender stereotyping in sex discrimination. Psychology, Public Policy and Law, 5, 655-692.

Castillo-Mayén, M. R. y Montes-Berges, B. (2007). Escala de estereotipos de género actuales. Iniciación a la Investigación, 2:a5. Recuperado de yor diversidad con que las mujeres son definidas en la actualidad en comparación con los hombres. Así, en nuestra sociedad podemos observar que los roles sociales considerados femeninos continúan siendo desempeñados principalmente por las mujeres, mientras que los roles sociales considerados masculinos son ocupados mayoritariamente por los hombres, pero también por las mujeres. De esta manera, en consonancia con los postulados de la teoría del rol social, al observar que las mujeres llevan a cabo una amplia variedad de roles, las características estereotípicas que se le asignan pueden estar más sujetas a las diferencias que expresan las personas según sus características sociodemográficas.

Sin embargo, aunque se han recogido numerosos cambios en los estereotipos de género, los resultados de este estudio también han puesto de manifiesto la vigencia de un amplio número de estereotipos tradicionales (ej., sumisas e inseguras para las mujeres, egoístas e insensibles para los hombres). Partiendo de que los estereotipos en general, y los estereotipos de género en particular, subyacen a los fenómenos discriminatorios, el patrón de resultados obtenido posiblemente esté reflejando lo que sucede en nuestra sociedad, pues si bien algunos procesos y circunstancias de las relaciones intergrupales entre los grupos de género están evolucionando favorablemente y se encuentran en proceso de cambio, otros aspectos se mantienen arraigados y son más resistentes. Entre los procesos y circunstancias que se están modificando encontramos un menor apoyo explícito a las actitudes sexistas y un mayor respaldo legal e institucional para la igualdad entre mujeres y hombres, así como una mayor presencia de las mujeres en niveles educativos superiores y en la ocupación de algunos puestos de liderazgo. Sin embargo, la persistente transmisión de los estereotipos y roles de género a través de los medios de comunicación y de otros agentes de socialización contribuye a que tales características sigan impregnadas en nuestra cultura, manifestándose en las distintas circunstancias en que las mujeres se siguen encontrando desfavorecidas, y donde la violencia contra ellas es el máximo exponente.

Agradecimientos.- Las autoras quieren agradecer al Dr. Ricardo Segurado del Center for Support and Training in Analysis and Research (CSTAR) de la University College Dublin por su inestimable contribución en la corrección de los aspectos metodológicos sugeridos por los revisores, y a Dña. Izaskun Urruchi-Pérez por su apreciada labor en la revisión de la versión en inglés de este artículo. También desean agradecer a los revisores anónimos sus comentarios y sugerencias para mejorar este trabajo. http:/ / revistaselectronicas.ujaen.es/index.php/ininv/article/view/198/ 179

Diekman, A. B. y Eagly, A. H. (2000). Stereotypes as dynamic constructs: Women and men of the past, present, and future. Personality and Social Psychology Bulletin, 26, 1171-1188.

Diekman, A. B., Eagly, A. H., Mladinic, A. y Ferreira, M. C. (2005). Dynamic stereotypes about women and men in Latin America and the United States. Journal of Cross-Cultural Psychology, 36(2), 209-226. 
Dovidio, J. F., Evans, N. y Tyler, R. B. (1986). Racial stereotypes: the contents of their cognitive representation. Journal of Experimental Social Psychology, 22, 22-37.

Eagly, A. H. (1987). Sex differences in social behavior: A social-role interpretation. Hillsdale, NJ: Lawrence Erlbaum.

Eagly, A. H. y Mladinic, A. (1989). Gender stereotypes and attitudes toward women and men. Personality and Social Psychology Bulletin, 15(4), 543-558.

Eagly, A. H. y Mladinic, A. (1993). Are people prejudiced against women? Some answers from research on attitudes, gender stereotypes, and judgment of competence. En W. Stroebe y M. Hewstone (Eds.), European Review of Social Psychology (Vol. 5, pp. 1-35). Nueva York: Wiley.

Eagly, A. H., Wood, W. y Diekman, A. B. (2000). Social role theory of sex differences and similarities: A current appraisal. En T. Eckes y H. M. Trautner (Eds.), The developmental social psychology of gender (pp. 123-174). Mahwah, NJ: Lawrence Erlbaum.

Eagly, A. H., Wood, W. y Johannesen-Schmidt, M. C. (2004). Social role theory of sex differences and similarities: Implications for the partner preferences of women and men. En A. H. Eagly, A. E. Beall y R. J. Sternberg (Eds.), The Psychology of Gender (2a ed., pp. 269-295). Nueva York: Guilford.

Fiske, S. T. y Stevens, L. E. (1993). What's so special about sex? Gender stereotyping and discrimination. En S. Oskamp y M. Costanzo (Eds.), Gender issues in contemporary society (pp. 173-196). Thousand Oaks, CA: Sage.

García-Retamero, R., Müller. S. M. y López-Zafra, E. (2009). Sobre la maleabilidad de los estereotipos de género: influencia del tamaño de la población y el paso del tiempo en la percepción de hombres y mujeres. En J. Tous y Pallarès y J. M. Fabra Sopeña (Eds.), Actas del XI Congreso Nacional de Psicología Social, (Vol. 2, pp. 151-155). Tarragona: Universitat Rovira i Virgili.

Garrido Lora, M. (2007). Los estereotipos de género en la publicidad. Creatividad y Sociedad, 11, 53-71.

Glick, P. y Fiske, S. T. (2001). An ambivalent alliance: Hostile and benevolent sexism as complementary justifications for gender inequality. American Psychologist, 56, 109-118.

Heilman, M. E. (2001). Description and prescription: How gender stereotypes prevent women's ascent up the organizational ladder. Journal of Social Issues, 57, 657-674.

Hoffman, C. y Hurst, N. (1990). Gender stereotypes: Perception or rationalization? Journal of Personality and Social Psychology, 58, 197-208.

Jackman, M. R. (1994). The velvet glove: Paternalism and conflict in gender, class, and race relations. Berkeley, CA: University of California Press.

Jost, J. T. y Hamilton, D. L. (2005). Stereotypes in our culture. En J. F. Dovidio, P. Glick y L. A. Rudman (Eds.), On the nature of prejudice: Fifty years after Allport (pp. 208-224). Oxford: Blackwell.

Langford, T. y MacKinnon, N. J. (2000). The affective basis for the gendering of traits: Comparing the United States and Canada. Social Psychology Ouarterly, 63, 34-48.

López-Sáez, M. (2008). Estereotipos de género. En E. López-Zafra (Comp.), Mujer y lider: Cómo encontrar el camino en el laberinto del liderazgo con éxito (pp. 76-90). Jaén: Del Lunar.

López-Sáez, M. y Lisbona, A. (2009). Descriptive and prescriptive features of gender stereotyping. Relationships among its components. Revista de Psicología Social, 24(3), 363-379.

López-Sáez, M. y Morales, J. F. (1995). Gender stereotypes in the Spanish population: Looking toward the future. En L. Amâncio y C. Nogueira (Eds.), Gender, management and science (pp. 151-168). Braga: Instituto de Educaçâo e Psicologia, Universidade do Minho.
López-Sáez, M., Morales, J. F. y Lisbona, A. (2008). Evolution of gender stereotypes in Spain: Traits and roles. Spanish Journal of Psychology, 11(2), 609-617.

López-Zafra, E., García-Retamero, R., Diekman, A. y Eagly, A. H. (2008). Dinámica de estereotipos de género y poder: un estudio transcultural. Revista de Psicología Social, 23(2), 213-219.

Montes-Berges, B. (2002). Discriminación de género en situaciones de competición y cooperación con priming subliminal. Tesis doctoral no publicada. Universidad de Granada.

Montes-Berges, B. (2010, septiembre). The prediction of gender stereotypes' assignment and discriminatory behaviour to male and female nurses depending on social dominance levels. Comunicación presentada en la Annual Conference of The British Psychological Society, Social Psychology Section, Winchester, Reino Unido.

Montilla, G., Aranda, M. y Montes-Berges, B. (2010). Perception of battered women with priming experimentation. En M. C. Castellanos, A. Marful y T. Bajo (Eds.), 8th SEPEX Conference. 1st joint Conference of the EPS and SEPEX. Book of abstracts (p. 177). Granada: Sider.

Morales J. F. y López-Sáez, M. (1993). Bases para la construcción de un sistema de indicadores sociales de estereotipia de género. Psicothema, 5, 123-132.

Moya, M. (2003). El análisis psicosocial del género. En J. F. Morales y C. Huici (Eds.), Estudios de Psicología Social (pp. 175-221). Madrid: UNED.

Moya, M. y Pérez, C. (1990). Nuevas perspectivas en el estudio de los estereotipos de género. En P. Valcárcel y J. L. Meliá (Eds.), Métodos y técnicas de intervención (pp. 49-60). Barcelona: Promociones y Publicaciones Universitarias.

Moya, M. y Puertas, S. (2003). Definición de sexismo y conceptos relacionados. En D. Páez, I. Fernández, S. Ubillos y E. Zubieta (Coords.), Psicología Social, Cultura y Educación (pp. 208-223). Madrid: Prentice Hall.

Rocha-Sánchez, T. E. y Díaz-Loving, R. (2005). Cultura de género: la brecha ideológica entre hombres y mujeres. Anales de Psicología, 21(1), 42-49.

Sánchez-Herrero Arbide, S., Sánchez-López, M. P. y Dresch, V. (2009). Hombres y trabajo doméstico: variables demográficas, salud y satisfacción. Anales de Psicología, 25(2), 299-307.

Sczesny, S., Bosak, J., Diekman, A. B. y Twenge, J. M. (2008). Dynamics of sex-role stereotypes. En Y. Kashima, K. Fiedler y P. Freytag (Eds.), Stereotype dynamics. Language-based approaches to the formation, maintenance, and the transformation of stereotypes (pp. 135-161). Nueva York: Lawrence Erlbaum.

Spence, J. T. y Buckner, C. E. (2000). Instrumental and expressive traits, trait stereotypes, and sexist attitudes. Psychology of Women Quarterly, 24, 44-62.

Spence, J. T., Helmreich, R. L. y Stapp, J. (1974). The Personal Attributes Questionnaire: A measure of sex role stereotypes and masculinityfemininity. JSAS Catalog of Selected Documents in Psychology, 4, 43-44.

Steele, C. M. (1997). A threat in the air: How stereotypes shape intellectual identity and performance. American Psychologist, 52(6), 613-629.

Velasco Sacristán, M. S. (2003). Publicidad y Género: propuesta, diseño y aplicación de un modelo de análisis de las metáforas de género en la publicidad impresa en lengua inglesa. Tesis doctoral, Universidad de Valladolid. Alicante: Biblioteca Virtual Miguel de Cervantes.

Wilde, A. y Diekman, A. B. (2005). Cross-cultural similarities and differences in dynamic stereotypes: A comparison between Germany and the United States. Psychology of Women Ouarterly, 29(2), 188-196.

Williams, J. E. y Best, D. L. (1982). Measuring sex stereotypes: A thirty nation $s t u d y$. Berkeley, CA: Sage.

Williams, J. E. y Best, D. L. (1990). Measuring sex stereotypes: A multi-nation study. Newbury Park, CA: Sage.

(Articulo recibido: 5-11-2011; revisado: 24-1-2013, aceptado: 28-10-2013) 


\section{Anexo 1. Escala de Características Estereotípicas (versión mujeres) ${ }^{4}$}

Desde el Departamento de Psicología de la Universidad de Jaén, estamos realizando un estudio para el cual es importante conocer su opinión sobre cómo cree usted que las mujeres en general son vistas por la sociedad actualmente. Por ello le pedimos que, por favor, exprese en qué grado cree que los adjetivos presentados a continuación definen, actualmente en nuestra sociedad, a las mujeres en general. Para ello, utilice la siguiente escala, señalando con un círculo un número, donde 1 indicaría que está totalmente en desacuerdo con que ese adjetivo define a las mujeres según nuestra sociedad actual, y 7 indicaría que está totalmente de acuerdo con que ese adjetivo define a las mujeres según nuestra sociedad actual, expresando los demás números los grados intermedios correspondientes. De este modo, 2 indicaría que está bastante en $\underline{\text { desacuerdo }}, \underline{3}$ que está en desacuerdo, $\underline{4}$ que no está ni de acuerdo ni en desacuerdo, $\underline{5}$ que está de acuerdo, y $\underline{6}$ que está bastante de acuerdo.

$\begin{array}{ccccccc}\begin{array}{c}\text { Totalmente en } \\ \text { desacuerdo }\end{array} & \begin{array}{c}\text { Bastante en } \\ \text { desacuerdo }\end{array} & \text { En desacuerdo } & \begin{array}{c}\text { Ni de acuerdo } \\ \text { ni en desacuerdo }\end{array} & \text { De acuerdo } & \begin{array}{c}\text { Bastante de } \\ \text { acuerdo }\end{array} & \begin{array}{c}\text { Totalmente de } \\ \text { acuerdo }\end{array} \\ 1 & 2 & 3 & 4 & 5 & 6 & 7\end{array}$

Actualmente, según la sociedad actual, las mujeres en general son vistas como:

\begin{tabular}{|c|c|c|c|c|c|c|c|}
\hline & $\begin{array}{c}\text { Totalmente en } \\
\text { desacuerdo }\end{array}$ & $\begin{array}{c}\text { Bastante en } \\
\text { desacuerdo }\end{array}$ & $\begin{array}{c}\text { En } \\
\text { desacuerdo }\end{array}$ & $\begin{array}{l}\text { Ni de acuerdo ni } \\
\text { en desacuerdo }\end{array}$ & De acuerdo & $\begin{array}{c}\text { Bastante de } \\
\text { acuerdo }\end{array}$ & $\begin{array}{l}\text { Totalmente de } \\
\text { acuerdo }\end{array}$ \\
\hline Autoeficaces & 1 & 2 & 3 & 4 & 5 & 6 & 7 \\
\hline Cordiales & 1 & 2 & 3 & 4 & 5 & 6 & 7 \\
\hline Competentes & 1 & 2 & 3 & 4 & 5 & 6 & 7 \\
\hline Orientadas a la familia & 1 & 2 & 3 & 4 & 5 & 6 & 7 \\
\hline Con alta habilidad verbal & 1 & 2 & 3 & 4 & 5 & 6 & 7 \\
\hline Cariñosas & 1 & 2 & 3 & 4 & 5 & 6 & 7 \\
\hline $\begin{array}{l}\text { Piensan que las relaciones humanas } \\
\text { son importantes }\end{array}$ & 1 & 2 & 3 & 4 & 5 & 6 & 7 \\
\hline Exigentes & 1 & 2 & 3 & 4 & 5 & 6 & 7 \\
\hline Complicadas & 1 & 2 & 3 & 4 & 5 & 6 & 7 \\
\hline Habladoras & 1 & 2 & 3 & 4 & 5 & 6 & 7 \\
\hline Miedosas & 1 & 2 & 3 & 4 & 5 & 6 & 7 \\
\hline Volubles & 1 & 2 & 3 & 4 & 5 & 6 & 7 \\
\hline Imprevisibles & 1 & 2 & 3 & 4 & 5 & 6 & 7 \\
\hline Científicas & 1 & 2 & 3 & 4 & 5 & 6 & 7 \\
\hline Orientadas a la carrera profesional & 1 & 2 & 3 & 4 & 5 & 6 & 7 \\
\hline Autónomas & 1 & 2 & 3 & 4 & 5 & 6 & 7 \\
\hline Con alta habilidad en matemáticas & 1 & 2 & 3 & 4 & 5 & 6 & 7 \\
\hline Egoístas & 1 & 2 & 3 & 4 & 5 & 6 & 7 \\
\hline Fanfarronas & 1 & 2 & 3 & 4 & 5 & 6 & 7 \\
\hline Groseras & 1 & 2 & 3 & 4 & 5 & 6 & 7 \\
\hline Perezosas & 1 & 2 & 3 & 4 & 5 & 6 & 7 \\
\hline Frívolas & 1 & 2 & 3 & 4 & 5 & 6 & 7 \\
\hline Narcisistas & 1 & 2 & 3 & 4 & 5 & 6 & 7 \\
\hline Dependientes & 1 & 2 & 3 & 4 & 5 & 6 & 7 \\
\hline Afectivas & 1 & 2 & 3 & 4 & 5 & 6 & 7 \\
\hline Emocionales & 1 & 2 & 3 & 4 & 5 & 6 & 7 \\
\hline Valientes & 1 & 2 & 3 & 4 & 5 & 6 & 7 \\
\hline Decididas & 1 & 2 & 3 & 4 & 5 & 6 & 7 \\
\hline Destinadas a la reproducción & 1 & 2 & 3 & 4 & 5 & 6 & 7 \\
\hline Competitivas & 1 & 2 & 3 & 4 & 5 & 6 & 7 \\
\hline Sociables & 1 & 2 & 3 & 4 & 5 & 6 & 7 \\
\hline Independientes & 1 & 2 & 3 & 4 & 5 & 6 & 7 \\
\hline Supersticiosas & 1 & 2 & 3 & 4 & 5 & 6 & 7 \\
\hline
\end{tabular}

\footnotetext{
${ }^{4}$ En este anexo sólo se presenta la versión utilizada para conocer las características estereotípicas asociadas a las mujeres. La versión utilizada para conocer las características asociadas a los hombres contemplaba las adaptaciones correspondientes de las instrucciones y de la terminación de los adjetivos.
} 


\begin{tabular}{|c|c|c|c|c|c|c|c|}
\hline & \begin{tabular}{|c|}
$\begin{array}{c}\text { Totalmente en } \\
\text { desacuerdo }\end{array}$ \\
\end{tabular} & \begin{tabular}{|l|}
$\begin{array}{l}\text { Bastante en } \\
\text { desacuerdo }\end{array}$ \\
\end{tabular} & $\begin{array}{c}\text { En } \\
\text { desacuerdo }\end{array}$ & \begin{tabular}{|c|} 
Ni de acuerdo \\
ni en desacuerdo
\end{tabular} & De acuerdo & $\begin{array}{c}\text { Bastante de } \\
\text { acuerdo }\end{array}$ & $\begin{array}{c}\text { Totalmente de } \\
\text { acuerdo }\end{array}$ \\
\hline Cálidas & 1 & 2 & 3 & 4 & 5 & 6 & 7 \\
\hline Vengativas & 1 & 2 & 3 & 4 & 5 & 6 & 7 \\
\hline Comprensivas & 1 & 2 & 3 & 4 & 5 & 6 & 7 \\
\hline Celosas & 1 & 2 & 3 & 4 & 5 & 6 & 7 \\
\hline Misteriosas & 1 & 2 & 3 & 4 & 5 & 6 & 7 \\
\hline Sentimentales & 1 & 2 & 3 & 4 & 5 & 6 & 7 \\
\hline Arriesgadas & 1 & 2 & 3 & 4 & 5 & 6 & 7 \\
\hline Destinadas al placer sexual & 1 & 2 & 3 & 4 & 5 & 6 & 7 \\
\hline Profesionales & 1 & 2 & 3 & 4 & 5 & 6 & 7 \\
\hline Detallistas & 1 & 2 & 3 & 4 & 5 & 6 & 7 \\
\hline Honradas & 1 & 2 & 3 & 4 & 5 & 6 & 7 \\
\hline Temperamentales & 1 & 2 & 3 & 4 & 5 & 6 & 7 \\
\hline Calculadoras & 1 & 2 & 3 & 4 & 5 & 6 & 7 \\
\hline Atentas & 1 & 2 & 3 & 4 & 5 & 6 & 7 \\
\hline Maduras & 1 & 2 & 3 & 4 & 5 & 6 & 7 \\
\hline \begin{tabular}{|l} 
Progresistas \\
\end{tabular} & 1 & 2 & 3 & 4 & 5 & 6 & 7 \\
\hline Depresivas & 1 & 2 & 3 & 4 & 5 & 6 & 7 \\
\hline Capaces & 1 & 2 & 3 & 4 & 5 & 6 & 7 \\
\hline Tacañas & 1 & 2 & 3 & 4 & 5 & 6 & 7 \\
\hline Preocupadas por el bienestar de los demás & 1 & 2 & 3 & 4 & 5 & 6 & 7 \\
\hline Eficientes & 1 & 2 & 3 & 4 & 5 & 6 & 7 \\
\hline Lógicas & 1 & 2 & 3 & 4 & 5 & 6 & 7 \\
\hline Caprichosas & 1 & 2 & 3 & 4 & 5 & 6 & 7 \\
\hline Poco emocionales & 1 & 2 & 3 & 4 & 5 & 6 & 7 \\
\hline Emprendedoras & 1 & 2 & 3 & 4 & 5 & 6 & 7 \\
\hline Solitarias & 1 & 2 & 3 & 4 & 5 & 6 & 7 \\
\hline Impulsivas & 1 & 2 & 3 & 4 & 5 & 6 & 7 \\
\hline Charlatanas & 1 & 2 & 3 & 4 & 5 & 6 & 7 \\
\hline Maleducadas & 1 & 2 & 3 & 4 & 5 & 6 & 7 \\
\hline Líderes & 1 & 2 & 3 & 4 & 5 & 6 & 7 \\
\hline Fieles & 1 & 2 & 3 & 4 & 5 & 6 & 7 \\
\hline Nerviosas & 1 & 2 & 3 & 4 & 5 & 6 & 7 \\
\hline Cercanas & 1 & 2 & 3 & 4 & 5 & 6 & 7 \\
\hline Histéricas & 1 & 2 & 3 & 4 & 5 & 6 & 7 \\
\hline Viciosas & 1 & 2 & 3 & 4 & 5 & 6 & 7 \\
\hline Dóciles & 1 & 2 & 3 & 4 & 5 & 6 & 7 \\
\hline Incompletas & 1 & 2 & 3 & 4 & 5 & 6 & 7 \\
\hline Conflictivas & 1 & 2 & 3 & 4 & 5 & 6 & 7 \\
\hline Rebeldes & 1 & 2 & 3 & 4 & 5 & 6 & 7 \\
\hline Cuidadoras & 1 & 2 & 3 & 4 & 5 & 6 & 7 \\
\hline Quisquillosas & 1 & 2 & 3 & 4 & 5 & 6 & 7 \\
\hline Generosas & 1 & 2 & 3 & 4 & 5 & 6 & 7 \\
\hline Oprimidas & 1 & 2 & 3 & 4 & 5 & 6 & 7 \\
\hline Perfeccionistas & 1 & 2 & 3 & 4 & 5 & 6 & 7 \\
\hline Saludables & 1 & 2 & 3 & 4 & 5 & 6 & 7 \\
\hline Fuertes emocionalmente & 1 & 2 & 3 & 4 & 5 & 6 & 7 \\
\hline Complacientes & 1 & 2 & 3 & 4 & 5 & 6 & 7 \\
\hline Chistosas & 1 & 2 & 3 & 4 & 5 & 6 & 7 \\
\hline Bondadosas & 1 & 2 & 3 & 4 & 5 & 6 & 7 \\
\hline Cobardes & 1 & 2 & 3 & 4 & 5 & 6 & 7 \\
\hline Presumidas & 1 & 2 & 3 & 4 & 5 & 6 & 7 \\
\hline Encantadoras & 1 & 2 & 3 & 4 & 5 & 6 & 7 \\
\hline \begin{tabular}{|l} 
Delicadas \\
\end{tabular} & 1 & 2 & 3 & 4 & 5 & 6 & 7 \\
\hline Sumisas & 1 & 2 & 3 & 4 & 5 & 6 & 7 \\
\hline Románticas & 1 & 2 & 3 & 4 & 5 & 6 & 7 \\
\hline Sufridas & 1 & 2 & 3 & 4 & 5 & 6 & 7 \\
\hline Elegantes & 1 & 2 & 3 & 4 & 5 & 6 & 7 \\
\hline Autosuficientes & 1 & 2 & 3 & 4 & 5 & 6 & 7 \\
\hline Pesimistas & 1 & 2 & 3 & 4 & 5 & 6 & 7 \\
\hline
\end{tabular}




\begin{tabular}{|c|c|c|c|c|c|c|c|}
\hline & \begin{tabular}{|c|}
$\begin{array}{c}\text { Totalmente } \\
\text { en desacuerdo }\end{array}$ \\
\end{tabular} & $\begin{array}{l}\text { Bastante en } \\
\text { desacuerdo }\end{array}$ & \begin{tabular}{|c|} 
En \\
desacuerdo
\end{tabular} & \begin{tabular}{|c|} 
Ni de acuerdo \\
ni en desacuerdo
\end{tabular} & De acuerdo & \begin{tabular}{|c|}
$\begin{array}{c}\text { Bastante de } \\
\text { acuerdo }\end{array}$ \\
\end{tabular} & $\begin{array}{c}\text { Totalmente de } \\
\text { acuerdo }\end{array}$ \\
\hline Ingeniosas & 1 & 2 & 3 & 4 & 5 & 6 & 7 \\
\hline Educadas & 1 & 2 & 3 & 4 & 5 & 6 & 7 \\
\hline Obsesivas & 1 & 2 & 3 & 4 & 5 & 6 & 7 \\
\hline Espontáneas & 1 & 2 & 3 & 4 & 5 & 6 & 7 \\
\hline Severas & 1 & 2 & 3 & 4 & 5 & 6 & 7 \\
\hline Cuidadosas & 1 & 2 & 3 & 4 & 5 & 6 & 7 \\
\hline Sencillas & 1 & 2 & 3 & 4 & 5 & 6 & 7 \\
\hline Trabajadoras & 1 & 2 & 3 & 4 & 5 & 6 & 7 \\
\hline Tranquilas & 1 & 2 & 3 & 4 & 5 & 6 & 7 \\
\hline Naturales & 1 & 2 & 3 & 4 & 5 & 6 & 7 \\
\hline Inventivas (de inventar) & 1 & 2 & 3 & 4 & 5 & 6 & 7 \\
\hline Sabelotodo & 1 & 2 & 3 & 4 & 5 & 6 & 7 \\
\hline Actúan con franqueza & 1 & 2 & 3 & 4 & 5 & 6 & 7 \\
\hline Hipócritas & 1 & 2 & 3 & 4 & 5 & 6 & 7 \\
\hline Intuitivas & 1 & 2 & 3 & 4 & 5 & 6 & 7 \\
\hline Puras & 1 & 2 & 3 & 4 & 5 & 6 & 7 \\
\hline Enrevesadas & 1 & 2 & 3 & 4 & 5 & 6 & 7 \\
\hline Distantes & 1 & 2 & 3 & 4 & 5 & 6 & 7 \\
\hline Sexualmente activas & 1 & 2 & 3 & 4 & 5 & 6 & 7 \\
\hline Amantes de los/las niños/as & 1 & 2 & 3 & 4 & 5 & 6 & 7 \\
\hline Compasivas & 1 & 2 & 3 & 4 & 5 & 6 & 7 \\
\hline Sinceras & 1 & 2 & 3 & 4 & 5 & 6 & 7 \\
\hline Rápidas & 1 & 2 & 3 & 4 & 5 & 6 & 7 \\
\hline Modernas & 1 & 2 & 3 & 4 & 5 & 6 & 7 \\
\hline Triunfadoras & 1 & 2 & 3 & 4 & 5 & 6 & 7 \\
\hline Con carácter & 1 & 2 & 3 & 4 & 5 & 6 & 7 \\
\hline Consideradas & 1 & 2 & 3 & 4 & 5 & 6 & 7 \\
\hline Despreciables & 1 & 2 & 3 & 4 & 5 & 6 & 7 \\
\hline Estúpidas & 1 & 2 & 3 & 4 & 5 & 6 & 7 \\
\hline Amenazantes & 1 & 2 & 3 & 4 & 5 & 6 & 7 \\
\hline Inteligentes & 1 & 2 & 3 & 4 & 5 & 6 & 7 \\
\hline Orientadas hacia la autorrealización & 1 & 2 & 3 & 4 & 5 & 6 & 7 \\
\hline De alto estatus & 1 & 2 & 3 & 4 & 5 & 6 & 7 \\
\hline Limpias & 1 & 2 & 3 & 4 & 5 & 6 & 7 \\
\hline Insensibles & 1 & 2 & 3 & 4 & 5 & 6 & 7 \\
\hline Maltratadoras & 1 & 2 & 3 & 4 & 5 & 6 & 7 \\
\hline Respetables & 1 & 2 & 3 & 4 & 5 & 6 & 7 \\
\hline Nobles & 1 & 2 & 3 & 4 & 5 & 6 & 7 \\
\hline Sensatas & 1 & 2 & 3 & 4 & 5 & 6 & 7 \\
\hline Promiscuas & 1 & 2 & 3 & 4 & 5 & 6 & 7 \\
\hline Maniáticas & 1 & 2 & 3 & 4 & 5 & 6 & 7 \\
\hline Tercas & 1 & 2 & 3 & 4 & 5 & 6 & 7 \\
\hline Defectuosas & 1 & 2 & 3 & 4 & 5 & 6 & 7 \\
\hline Frías & 1 & 2 & 3 & 4 & 5 & 6 & 7 \\
\hline Buenas conductoras & 1 & 2 & 3 & 4 & 5 & 6 & 7 \\
\hline Conciliadoras & 1 & 2 & 3 & 4 & 5 & 6 & 7 \\
\hline Con buen gusto & 1 & 2 & 3 & 4 & 5 & 6 & 7 \\
\hline Infantiles & 1 & 2 & 3 & 4 & 5 & 6 & 7 \\
\hline Anteponen sus logros a los aspectos emocionales & 1 & 2 & 3 & 4 & 5 & 6 & 7 \\
\hline Mimadas & 1 & 2 & 3 & 4 & 5 & 6 & 7 \\
\hline Prácticas & 1 & 2 & 3 & 4 & 5 & 6 & 7 \\
\hline Impertinentes & 1 & 2 & 3 & 4 & 5 & 6 & 7 \\
\hline Graciosas & 1 & 2 & 3 & 4 & 5 & 6 & 7 \\
\hline Hogareñas & 1 & 2 & 3 & 4 & 5 & 6 & 7 \\
\hline Amables & 1 & 2 & 3 & 4 & 5 & 6 & 7 \\
\hline De bajo estatus & 1 & 2 & 3 & 4 & 5 & 6 & 7 \\
\hline Instructivas & 1 & 2 & 3 & 4 & 5 & 6 & 7 \\
\hline Coléricas & 1 & 2 & 3 & 4 & 5 & 6 & 7 \\
\hline
\end{tabular}




\begin{tabular}{|c|c|c|c|c|c|c|c|}
\hline & $\begin{array}{c}\text { Totalmente en } \\
\text { desacuerdo }\end{array}$ & $\begin{array}{l}\begin{array}{c}\text { Bastante en } \\
\text { desacuerdo }\end{array} \\
\end{array}$ & En desacuerdo & $\begin{array}{c}\text { Ni de acuerdo ni } \\
\text { en desacuerdo }\end{array}$ & De acuerdo & $\begin{array}{c}\text { Bastante de } \\
\text { acuerdo }\end{array}$ & $\begin{array}{c}\text { Totalmente de } \\
\text { acuerdo }\end{array}$ \\
\hline Optimistas & 1 & 2 & 3 & 4 & 5 & 6 & 7 \\
\hline Chismosas & 1 & 2 & 3 & 4 & 5 & 6 & 7 \\
\hline Débiles & 1 & 2 & 3 & 4 & 5 & 6 & 7 \\
\hline Libres & 1 & 2 & 3 & 4 & 5 & 6 & 7 \\
\hline Amigables & 1 & 2 & 3 & 4 & 5 & 6 & 7 \\
\hline Liberadas & 1 & 2 & 3 & 4 & 5 & 6 & 7 \\
\hline \begin{tabular}{|l} 
Habilidosas \\
\end{tabular} & 1 & 2 & 3 & 4 & 5 & 6 & 7 \\
\hline Dulces & 1 & 2 & 3 & 4 & 5 & 6 & 7 \\
\hline Conservadoras & 1 & 2 & 3 & 4 & 5 & 6 & 7 \\
\hline Humildes & 1 & 2 & 3 & 4 & 5 & 6 & 7 \\
\hline Inútiles & 1 & 2 & 3 & 4 & 5 & 6 & 7 \\
\hline Tiernas & 1 & 2 & 3 & 4 & 5 & 6 & 7 \\
\hline Reservadas & 1 & 2 & 3 & 4 & 5 & 6 & 7 \\
\hline Buenas & 1 & 2 & 3 & 4 & 5 & 6 & 7 \\
\hline Frustradas & 1 & 2 & 3 & 4 & 5 & 6 & 7 \\
\hline Tenaces & 1 & 2 & 3 & 4 & 5 & 6 & 7 \\
\hline Honestas & 1 & 2 & 3 & 4 & 5 & 6 & 7 \\
\hline Paranoicas & 1 & 2 & 3 & 4 & 5 & 6 & 7 \\
\hline Manipuladoras & 1 & 2 & 3 & 4 & 5 & 6 & 7 \\
\hline \begin{tabular}{|l|} 
Despreocupadas \\
\end{tabular} & 1 & 2 & 3 & 4 & 5 & 6 & 7 \\
\hline Confiadas & 1 & 2 & 3 & 4 & 5 & 6 & 7 \\
\hline Asertivas & 1 & 2 & 3 & 4 & 5 & 6 & 7 \\
\hline Dominadas & 1 & 2 & 3 & 4 & 5 & 6 & 7 \\
\hline Orientadas hacia el logro personal & 1 & 2 & 3 & 4 & 5 & 6 & 7 \\
\hline Empáticas & 1 & 2 & 3 & 4 & 5 & 6 & 7 \\
\hline Respetuosas & 1 & 2 & 3 & 4 & 5 & 6 & 7 \\
\hline Fuertes físicamente & 1 & 2 & 3 & 4 & 5 & 6 & 7 \\
\hline Peligrosas & 1 & 2 & 3 & 4 & 5 & 6 & 7 \\
\hline Confidentes & 1 & 2 & 3 & 4 & 5 & 6 & 7 \\
\hline Pacíficas & 1 & 2 & 3 & 4 & 5 & 6 & 7 \\
\hline Con éxito profesional & 1 & 2 & 3 & 4 & 5 & 6 & 7 \\
\hline Leales & 1 & 2 & 3 & 4 & 5 & 6 & 7 \\
\hline Amantes del peligro & 1 & 2 & 3 & 4 & 5 & 6 & 7 \\
\hline Duras & 1 & 2 & 3 & 4 & 5 & 6 & 7 \\
\hline Minuciosas & 1 & 2 & 3 & 4 & 5 & 6 & 7 \\
\hline Coquetas & 1 & 2 & 3 & 4 & 5 & 6 & 7 \\
\hline Desquiciadas & 1 & 2 & 3 & 4 & 5 & 6 & 7 \\
\hline Creativas & 1 & 2 & 3 & 4 & 5 & 6 & 7 \\
\hline Mentirosas & 1 & 2 & 3 & 4 & 5 & 6 & 7 \\
\hline Afectuosas & 1 & 2 & 3 & 4 & 5 & 6 & 7 \\
\hline Pacientes & 1 & 2 & 3 & 4 & 5 & 6 & 7 \\
\hline Superficiales & 1 & 2 & 3 & 4 & 5 & 6 & 7 \\
\hline Religiosas & 1 & 2 & 3 & 4 & 5 & 6 & 7 \\
\hline Bordes & 1 & 2 & 3 & 4 & 5 & 6 & 7 \\
\hline Atractivas & 1 & 2 & 3 & 4 & 5 & 6 & 7 \\
\hline \begin{tabular}{|l|} 
Realistas \\
\end{tabular} & 1 & 2 & 3 & 4 & 5 & 6 & 7 \\
\hline Simpáticas & 1 & 2 & 3 & 4 & 5 & 6 & 7 \\
\hline \begin{tabular}{|l|} 
Infieles \\
\end{tabular} & 1 & 2 & 3 & 4 & 5 & 6 & 7 \\
\hline Poderosas & 1 & 2 & 3 & 4 & 5 & 6 & 7 \\
\hline Desagradables & 1 & 2 & 3 & 4 & 5 & 6 & 7 \\
\hline Eficaces & 1 & 2 & 3 & 4 & 5 & 6 & 7 \\
\hline Idealistas & 1 & 2 & 3 & 4 & 5 & 6 & 7 \\
\hline Falsas & 1 & 2 & 3 & 4 & 5 & 6 & 7 \\
\hline Imprudentes & 1 & 2 & 3 & 4 & 5 & 6 & 7 \\
\hline Materialistas & 1 & 2 & 3 & 4 & 5 & 6 & 7 \\
\hline Lentas & 1 & 2 & 3 & 4 & 5 & 6 & 7 \\
\hline Expresivas & 1 & 2 & 3 & 4 & 5 & 6 & 7 \\
\hline Familiares & 1 & 2 & 3 & 4 & 5 & 6 & 7 \\
\hline \begin{tabular}{|l|} 
Ingenuas \\
\end{tabular} & 1 & 2 & 3 & 4 & 5 & 6 & 7 \\
\hline \begin{tabular}{|l|} 
Activas \\
\end{tabular} & 1 & 2 & 3 & 4 & 5 & 6 & 7 \\
\hline Lloronas & 1 & 2 & 3 & 4 & 5 & 6 & 7 \\
\hline
\end{tabular}




\begin{tabular}{|c|c|c|c|c|c|c|c|}
\hline & $\begin{array}{c}\begin{array}{c}\text { Totalmente en } \\
\text { desacuerdo }\end{array} \\
\text {. }\end{array}$ & $\begin{array}{l}\text { Bastante en } \\
\text { desacuerdo }\end{array}$ & $\begin{array}{c}\text { En } \\
\text { desacuerdo }\end{array}$ & $\begin{array}{l}\text { Ni de acuerdo ni } \\
\text { en desacuerdo }\end{array}$ & De acuerdo & $\begin{array}{l}\text { Bastante de } \\
\text { acuerdo }\end{array}$ & $\begin{array}{l}\text { Totalmente de } \\
\text { acuerdo }\end{array}$ \\
\hline \begin{tabular}{|l|} 
Serviciales \\
\end{tabular} & 1 & 2 & 3 & 4 & 5 & 6 & 7 \\
\hline \begin{tabular}{|l|} 
Conquistadoras \\
\end{tabular} & 1 & 2 & 3 & 4 & 5 & 6 & 7 \\
\hline Dominantes & 1 & 2 & 3 & 4 & 5 & 6 & 7 \\
\hline \begin{tabular}{|l} 
Juerguistas \\
\end{tabular} & 1 & 2 & 3 & 4 & 5 & 6 & 7 \\
\hline Firmes & 1 & 2 & 3 & 4 & 5 & 6 & 7 \\
\hline Artistas & 1 & 2 & 3 & 4 & 5 & 6 & 7 \\
\hline Orgullosas & 1 & 2 & 3 & 4 & 5 & 6 & 7 \\
\hline Soñadoras & 1 & 2 & 3 & 4 & 5 & 6 & 7 \\
\hline Curiosas & 1 & 2 & 3 & 4 & 5 & 6 & 7 \\
\hline Vanidosas & 1 & 2 & 3 & 4 & 5 & 6 & 7 \\
\hline \begin{tabular}{|l|} 
Protegidas \\
\end{tabular} & 1 & 2 & 3 & 4 & 5 & 6 & 7 \\
\hline Individualistas & 1 & 2 & 3 & 4 & 5 & 6 & 7 \\
\hline Cultas & 1 & 2 & 3 & 4 & 5 & 6 & 7 \\
\hline \begin{tabular}{|l} 
Solidarias \\
\end{tabular} & 1 & 2 & 3 & 4 & 5 & 6 & 7 \\
\hline \begin{tabular}{|l} 
Aduladoras \\
\end{tabular} & 1 & 2 & 3 & 4 & 5 & 6 & 7 \\
\hline Sensibles & 1 & 2 & 3 & 4 & 5 & 6 & 7 \\
\hline Protectoras & 1 & 2 & 3 & 4 & 5 & 6 & 7 \\
\hline Alegres & 1 & 2 & 3 & 4 & 5 & 6 & 7 \\
\hline Autoritarias & 1 & 2 & 3 & 4 & 5 & 6 & 7 \\
\hline Inquietas & 1 & 2 & 3 & 4 & 5 & 6 & 7 \\
\hline Furiosas & 1 & 2 & 3 & 4 & 5 & 6 & 7 \\
\hline \begin{tabular}{|l|} 
Pasivas \\
\end{tabular} & 1 & 2 & 3 & 4 & 5 & 6 & 7 \\
\hline \begin{tabular}{|l} 
Perspicaces \\
\end{tabular} & 1 & 2 & 3 & 4 & 5 & 6 & 7 \\
\hline Víctimas & 1 & 2 & 3 & 4 & 5 & 6 & 7 \\
\hline Igualitaristas & 1 & 2 & 3 & 4 & 5 & 6 & 7 \\
\hline \begin{tabular}{|l|} 
Violentas \\
\end{tabular} & 1 & 2 & 3 & 4 & 5 & 6 & 7 \\
\hline Frágiles & 1 & 2 & 3 & 4 & 5 & 6 & 7 \\
\hline Sucias & 1 & 2 & 3 & 4 & 5 & 6 & 7 \\
\hline Racionales & 1 & 2 & 3 & 4 & 5 & 6 & 7 \\
\hline Inexpresivas & 1 & 2 & 3 & 4 & 5 & 6 & 7 \\
\hline Ariscas & 1 & 2 & 3 & 4 & 5 & 6 & 7 \\
\hline Malas & 1 & 2 & 3 & 4 & 5 & 6 & 7 \\
\hline \begin{tabular}{|l|} 
Agresivas \\
\end{tabular} & 1 & 2 & 3 & 4 & 5 & 6 & 7 \\
\hline Astutas & 1 & 2 & 3 & 4 & 5 & 6 & 7 \\
\hline Ambiciosas & 1 & 2 & 3 & 4 & 5 & 6 & 7 \\
\hline \begin{tabular}{|l|} 
Rencorosas \\
\end{tabular} & 1 & 2 & 3 & 4 & 5 & 6 & 7 \\
\hline Sutiles & 1 & 2 & 3 & 4 & 5 & 6 & 7 \\
\hline Vulnerables & 1 & 2 & 3 & 4 & 5 & 6 & 7 \\
\hline Inseguras & 1 & 2 & 3 & 4 & 5 & 6 & 7 \\
\hline Egocéntricas & 1 & 2 & 3 & 4 & 5 & 6 & 7 \\
\hline \begin{tabular}{|l|} 
Agradables \\
\end{tabular} & 1 & 2 & 3 & 4 & 5 & 6 & 7 \\
\hline De personalidad fuerte & 1 & 2 & 3 & 4 & 5 & 6 & 7 \\
\hline Atléticas & 1 & 2 & 3 & 4 & 5 & 6 & 7 \\
\hline Torpes & 1 & 2 & 3 & 4 & 5 & 6 & 7 \\
\hline Moralistas & 1 & 2 & 3 & 4 & 5 & 6 & 7 \\
\hline $\begin{array}{l}\text { Destinadas al cuidado de } \\
\text { los/las hijos/as }\end{array}$ & 1 & 2 & 3 & 4 & 5 & 6 & 7 \\
\hline Incondicionales & 1 & 2 & 3 & 4 & 5 & 6 & 7 \\
\hline
\end{tabular}

Por favor, compruebe que ha respondido a todos los adjetivos.

¡iMUCHAS GRACIAS POR SU COLABORACIÓN!! 